\title{
REGULARITY OF ROOTS OF POLYNOMIALS
}

\author{
ADAM PARUSIŃSKI AND ARMIN RAINER
}

\begin{abstract}
We show that smooth curves of monic complex polynomials $P_{a}(Z)=Z^{n}+$ $\sum_{j=1}^{n} a_{j} Z^{n-j}, a_{j}: I \rightarrow \mathbb{C}$ with $I \subset \mathbb{R}$ a compact interval, have absolutely continuous roots in a uniform way. More precisely, there exists a positive integer $k$ and a rational number $p>1$, both depending only on the degree $n$, such that if $a_{j} \in C^{k}$ then any continuous choice of roots of $P_{a}$ is absolutely continuous with derivatives in $L^{q}$ for all $1 \leq q<p$, in a uniform way with respect to $\max _{j}\left\|a_{j}\right\|_{C^{k}}$. The uniformity allows us to deduce also a multiparameter version of this result. The proof is based on formulas for the roots of the universal polynomial $P_{a}$ in terms of its coefficients $a_{j}$ which we derive using resolution of singularities. For cubic polynomials we compute the formulas as well as bounds for $k$ and $p$ explicitly.
\end{abstract}

\section{Contents}

Introduction

Part 1. Absolute continuity of roots

1. Formulas for the roots

2. Function spaces

3. Absolute continuity of roots

4. Multiparameter families of polynomials

Part 2. Formulas for the roots. Proof of Theorem 1.6 19

5. Strategy of the proof 19

6. A characterization of principality $\quad 19$

7. Convexity 20

8. Splitting $\quad 22$

9. Proof of Theorem 1.6 24

Part 3. Example. Roots of cubic polynomials. 27

10. Statement of result. $\quad 27$

11. Resolution of the discriminant $\quad 27$.

References $\quad 30$

2010 Mathematics Subject Classification. 26C10, 26A46, 30C15, 32S45.

Key words and phrases. Perturbation of complex polynomials, absolute continuity of roots, $L^{p}$ regularity, resolution of singularities.

Supported by the Austrian Science Fund (FWF), Grants P 22218-N13 and P 26735-N25, and by ANR project STAAVF (ANR-2011 BS01 009). 


\section{INTRODUCTION}

This paper is dedicated to the solution of a basic problem in perturbation theory and differential analysis. Given a monic polynomial with smooth coefficients (or a matrix with smooth entries) it is natural to ask for the optimal regularity of the roots (or of the eigenvalues). For instance, this question arises in finding local solutions of partial differential equations with multiple characteristics.

In connection with the study of a class of pseudodifferential systems, Spagnolo [26] asked whether a smooth $\left(C^{\infty}\right)$ curve of monic complex polynomials admits a locally absolutely continuous parameterization of its roots. And if so, whether it is possible to choose the absolutely continuous roots uniformly with respect to the coefficients on compact subintervals. We answer these questions affirmatively and prove the following stronger result; see also Theorem 3.5. Our proof builds on the recent result of Ghisi and Gobbino [7] who found the optimal regularity of radicals of functions that we combine with the resolution of singularities.

Main Theorem. For every $n \in \mathbb{N}_{>0}$ there is $k=k(n) \in \mathbb{N}_{>0}$ and $p=p(n)>1$ such that the following holds. Let $I \subset \mathbb{R}$ be a compact interval and let

$$
P_{a(t)}(Z)=Z^{n}+\sum_{j=1}^{n} a_{j}(t) Z^{n-j} \in C^{k}(I)[Z]
$$

be a monic polynomial with coefficients $a_{j} \in C^{k}(I), j=1, \ldots, n$.

(1) Let $\lambda_{j} \in C^{0}(I), j=1, \ldots, n$, be a continuous parameterization of the roots of $P_{a}$ on $I$. Then the distributional derivative of each $\lambda_{j}$ in $I$ is a measurable function $\lambda_{j}^{\prime} \in L^{q}(I)$ for every $q \in[1, p)$. In particular, each $\lambda_{j} \in W^{1, q}(I)$ for every $q \in[1, p)$.

(2) This regularity of the roots is uniform. Let $\left\{P_{a_{\nu}} ; \nu \in \mathcal{N}\right\}$,

$$
P_{a_{\nu}(t)}(Z)=Z^{n}+\sum_{j=1}^{n} a_{\nu, j}(t) Z^{n-j} \in C^{k}(I)[Z], \nu \in \mathcal{N},
$$

be a family of curves of polynomials, indexed by $\nu$ in some set $\mathcal{N}$, so that the set of coefficients $\left\{a_{\nu, j} ; \nu \in \mathcal{N}, j=1, \ldots, n\right\}$ is bounded in $C^{k}(I)$. Then the set

$$
\left\{\lambda_{\nu} \in C^{0}(I) ; P_{a_{\nu}}\left(\lambda_{\nu}\right)=0 \text { on } I, \nu \in \mathcal{N}\right\}
$$

is bounded in $W^{1, q}(I)$ for every $q \in[1, p)$.

$L^{q}$ denotes the Lebesgue space and $W^{1, q}$ the Sobolev space with respect to Lebesgue measure. We want to stress the fact that a continuous curve of monic complex polynomials $P_{a(t)}, t \in \mathbb{R}$, allows for a continuous parameterization of its roots. This is no longer true if the parameter space has more than one dimension due to monodromy. For multiparameter families of polynomials we obtain the following result; see also Theorem 4.1.

Multiparameter Theorem. Let $k=k(n) \in \mathbb{N}_{>0}$ and $p=p(n)>1$ be as above. Let $U \subset \mathbb{R}^{m}$ be open and let $P_{a(x)}(Z) \in C^{k}(U)[Z]$ be a monic polynomial with coefficients $a_{j} \in C^{k}(U), j=1, \ldots, n$. 
(1) Let $\lambda \in C^{0}(V)$ represent a root of $P_{a}$, i.e., $P_{a}(\lambda)=0$, on a relatively compact open subset $V \Subset U$. Then the distributional gradient of $\lambda$ in $V$ is a measurable function $\nabla \lambda \in\left[L^{q}(V)\right]^{m}$ for every $q \in[1, p)$. In particular, $\lambda \in W^{1, q}(V)$ for every $q \in[1, p)$.

(2) The regularity of the roots is uniform. Let $\left\{P_{a_{\nu}} ; \nu \in \mathcal{N}\right\}$ be a family of polynomials, indexed by $\nu$ in some set $\mathcal{N}$, so that the set of coefficients $\left\{a_{\nu, j} ; \nu \in \mathcal{N}, j=1, \ldots, n\right\}$ is bounded in $C^{k}(U)$. Let $V \Subset U$. Then the set

$$
\left\{\lambda_{\nu} \in C^{0}(V) ; P_{a_{\nu}}\left(\lambda_{\nu}\right)=0 \text { on } V, \nu \in \mathcal{N}\right\}
$$

is bounded in $W^{1, q}(V)$ for every $q \in[1, p)$.

In 26] Spagnolo proved that the pseudodifferential $n \times n$ system

$$
u_{t}+i A\left(t, D_{x}\right) u+B\left(t, D_{x}\right) u=f(t, x), \quad(t, x) \in I \times U,
$$

where $A(t, \xi), B(t, \xi)$ are matrix symbols of order 1 and 0 , respectively, and $A(t, \xi)$ is homogeneous of degree 1 in $\xi$ for $|\xi| \geq 1$, is locally solvable in the Gevrey class $G^{s}$ for $1 \leq s \leq n /(n-1)$ and semi-globally solvable in $G^{s}$ for $1<s<n /(n-1)$ under the following assumptions: the eigenvalues of $A(t, \xi)$ admit a parameterization $\tau_{1}(t, \xi), \ldots, \tau_{n}(t, \xi)$ such that each $\tau_{j}(t, \xi)$ is absolutely continuous in $t$, uniformly with respect to $\xi$, i.e.,

$$
\left(\mathcal{A}_{1}\right) \quad\left|\partial_{t} \tau_{j}(t, \xi)\right| \leq \mu(t, \xi)\left(1+|\xi|^{2}\right)^{\frac{1}{2}}, \quad \text { with } \mu(, \xi) \text { equi-integrable on } I,
$$

and for each $\xi$ the imaginary parts of the $\tau_{j}(t, \xi)$ do not change sign for varying $t$ and $j$, i.e., $\left(\mathcal{A}_{2}\right) \quad \forall \xi \quad$ either $\operatorname{Im} \tau_{j}(t, \xi) \geq 0, \quad \forall t, j, \quad$ or $\operatorname{Im} \tau_{j}(t, \xi) \leq 0, \quad \forall t, j$.

Our Main Theorem implies that the Assumption $\left(\mathcal{A}_{1}\right)$ is automatically satisfied. Indeed, this follows by applying the Main Theorem to the characteristic polynomial of the matrix $\left(1+|\xi|^{2}\right)^{-\frac{1}{2}} A(t, \xi)$ and noting that the entries of $\left(1+|\xi|^{2}\right)^{-\frac{1}{2}} A(t, \xi)$ and its iterated partial derivatives with respect to $t$ are globally bounded in $\xi$, since $A(t, \xi)$ is a symbol of order 1 .

Spagnolo formulated the removal of Assumption $\left(\mathcal{A}_{1}\right)$ as an open problem in [26], p. 1122, and he successfully tackled the case of quadratic and cubic polynomials in [25]. Note that the problems of smoothly choosing roots of polynomials on one hand and eigenvalues of arbitrary quadratic matrices on the other hand are equivalent; whereas the perturbation theory for normal matrices is easier and allows for stronger results, cf. Rainer [24] and references therein.

We would like to remark that our result represents a complex analogue of Bronshtein's Theorem on the regularity of the roots of hyperbolic polynomials. A monic polynomial is called hyperbolic if all its roots are real. Bronshtein's Theorem, first proved in Bronshtein [4] and generalizing the classical Glaeser inequality [8], states that any continuous parameterization of the roots of a hyperbolic polynomial of degree $n$ with $C^{n-1,1}$ coefficients is locally Lipschitz. It plays a crucial role for weakly hyperbolic Cauchy problems. Different proofs appeared in Wakabayashi [28] and in Parusiński and Rainer [20].

In the absence of hyperbolicity the roots cannot fulfill a Lipschitz condition and in a certain sense absolute continuity is the best one can hope for; in fact the degree of summability $p$ tends to 1 as $n$ goes to $\infty$. The first result towards absolute continuity of the roots is probably Lemma 1 in Colombini, Jannelli, and Spagnolo [5] which states that for a real-valued nonnegative function $f$ of class $C^{k, \alpha}$ on a compact interval $I$, with $k \in \mathbb{N}_{\geq 1}$ and $0 \leq \alpha \leq 1$, the 
radical $f^{1 /(k+\alpha)}$ is absolutely continuous on $I$ and satisfies

$$
\left\|\left(f^{\frac{1}{k+\alpha}}\right)^{\prime}\right\|_{L^{1}(I)}^{k+\alpha} \leq C(k, \alpha, I)\|f\|_{C^{k, \alpha}(I)} .
$$

Tarama [27] extended this lemma to real-valued functions (not necessarily non-negative). A better summability for the weak partial derivatives of $f^{1 /(k+1)}$ was obtained by Colombini and Lerner [6] for non-negative $C^{k+1}$ functions $f$ of several real variables.

The case of radicals of functions was completely settled recently by Ghisi and Gobbino [7] by finding their optimal regularity. They showed that, if $f$ is a real-valued continuous function and there exists $g \in C^{k, \alpha}(I)$ so that $|f|^{k+\alpha}=|g|$ on $I$, then $f^{\prime} \in L_{w}^{p}(I)$, where $\frac{1}{p}+\frac{1}{k+\alpha}=1$, and

$$
\left\|f^{\prime}\right\|_{p, w, I} \leq C(k) \max \left\{\left[\operatorname{Höld}_{\alpha, I}\left(g^{(k)}\right)\right]^{\frac{1}{k+\alpha}}|I|^{\frac{1}{p}},\left\|g^{\prime}\right\|_{L^{\infty}(I)}^{\frac{1}{k+\alpha}}\right\} ;
$$

in particular, $f \in W^{1, q}(I)$ for every $q \in[1, p)$. Here $L_{w}^{p}(I)$ denotes the weak Lebesgue space equipped with the quasi-norm $\|f\|_{p, w, I}:=\sup _{r \geq 0}\left\{r \cdot \mathcal{L}^{1}(\{t \in I ;|f(t)|>r\})^{\frac{1}{p}}\right\}$, where $\mathcal{L}^{1}$ is the one dimensional Lebesgue measure. By Höld ${ }_{\alpha, I}\left(g^{(k)}\right)$ we mean the $\alpha$-Hölder constant of $g^{(k)}$ on $I$, and $|I|=\mathcal{L}^{1}(I)$ is the length of the interval $I$. Ghisi and Gobbino also provided examples that show that the assumptions as well as the conclusion in their theorem are best possible. We use this result in a substantial way.

The mentioned results all treat special cases, where the algebraic structure of the polynomials is very simple: the roots are either given by radicals or can be expressed by radicals (by Cardano's formulas). A different approach was pursued in Rainer [22], where no restrictions on the algebraic structure of the polynomial were imposed. Under the assumption that no two roots meet with infinite order of contact it was shown that the roots of a $C^{\infty}$ curve of monic polynomials are locally absolutely continuous. We also mention Rainer [23], where it was proved that the roots of a monic polynomial whose coefficients are functions in several variables that belong to any quasianalytic class satisfying some stability properties admit a parameterization by (special) functions of bounded variation.

Our proof of the Main Theorem is based on formulas for the roots of the universal monic polynomial $P_{a}$ in terms of its coefficients $a=\left(a_{1}, \ldots, a_{n}\right) \in \mathbb{C}^{n}$. The derivation of these formulas represents the third major result of this paper; see Theorem 1.6. Using Hironaka's resolution of singularities [11], we construct a tower of smooth principalizations

$$
M_{1}=\mathbb{C}^{n} \stackrel{\sigma_{2}}{\longleftarrow} M_{2} \stackrel{\sigma_{3,2}}{\longleftarrow} M_{3} \stackrel{\sigma_{4,3}}{\longleftarrow} \ldots \stackrel{\sigma_{n, n-1}}{\longleftarrow} M_{n}
$$

which successively principalize the generalized discriminant ideals $\mathscr{D}_{m} \subset \mathbb{C}[a], m=2, \ldots, n$, that encode the stratification of the space of polynomials by root multiplicity. In fact, the zero set of $\mathscr{D}_{m}$ is exactly the set of those $a \in \mathbb{C}^{n}$ for which $P_{a}$ has at most $m-1$ distinct roots. We show that, locally on $M_{n}$, the roots of the pulled back polynomial $P_{\sigma_{n}^{*}(a)}$ are given by rational linear combinations $\sum_{m=1}^{n} A_{m} \varphi_{m} \circ \sigma_{n, m}$ where

$$
\varphi_{m}=f_{m}^{\alpha_{m}} \psi_{m}\left(y_{m, 1}^{1 / q_{m}}, \ldots, y_{m, r_{m}}^{1 / q_{m}}, y_{m, r_{m}+1}, \ldots, y_{m, n}\right) .
$$

Here $\sigma_{m}=\sigma_{2} \circ \sigma_{3,2} \circ \cdots \circ \sigma_{m, m-1}, \sigma_{n, m}=\sigma_{m+1, m} \circ \cdots \circ \sigma_{n, n-1}, f_{m} \in \mathscr{D}_{m}$ is a local generator of $\sigma_{m}^{*}\left(\mathscr{D}_{m}\right), \psi_{m}$ is a convergent power series, $q_{m} \in \mathbb{N}_{\geq 1}, \alpha_{m} \in \frac{1}{q_{m}} \mathbb{N}_{\geq 1}$, and $\left(y_{m, i}\right)$ is a privileged system of local coordinates so that $f_{m}^{-1}(0)$ is given by $y_{m, 1} \cdots y_{m, r_{m}}=0$ (cf. Subsection 
1.3 and Definition 1.4). These formulas are obtained in a natural way by a consecutive factorization procedure of the pull-backs $P_{\sigma_{i}^{*}(a)}, i=2, \ldots, n$, so that each step contributes exactly to one summand. Thanks to these formulas we are able to reduce the problem to radicals of functions and use the result of Ghisi and Gobbino (cf. Lemma 3.4).

The paper is divided into three parts. The first part presents the three main results of this paper. Section 1 is devoted to the formulation of the result on the formulas for the roots; see Theorem 1.6. In Section 2 we mainly fix notation on function spaces. The Main Theorem 3.5 is proved in Section 3, assuming validity of Theorem 1.6, and the Multiparameter Theorem 4.1 is deduced in Section 4 .

The second part of the paper is dedicated to the proof of Theorem 1.6. The strategy of the proof is briefly outlined in Section 5. In Section 6] we find a convenient criterion of principality of the ideals $\mathscr{D}_{m}$. In Sections 7 and 8 we further develop the necessary tools utilized in the proof of Theorem 1.6 which is finally carried out in Section 9 ,

In the third part we illustrate our method of proof by discussing the case of cubic polynomials in detail. Here the resolution is explicit, and we can specify more precisely the degree of differentiability of the coefficients and the degree of summability of the derivative of the roots, namely $k(3)=6$ and $p(3)=6 / 5$.

Acknowledgement. Part of the work was done while the second author enjoyed the hospitality of the mathematics department at the university of Nice.

Notation and terminology. By a normal crossing we mean a function that is locally equivalent to a monomial, i.e. equals a monomial times an analytic unit. The zero set of an ideal $\mathscr{I}$ will be denoted by $V(\mathscr{I})$. For two real-valued functions $\varphi$ and $\psi$ we write $\varphi \sim \psi$ if there exists $C>0$ such that $\varphi \leq C \psi$ and $\psi \leq C \varphi$. By $\lceil x\rceil$ we denote the celling function, that is the least integer bigger than or equal to $x$.

\section{Part 1. Absolute continuity of roots}

\section{Formulas FOR THE ROOTS}

\subsection{Generalized discriminant ideals. Let}

$$
P_{a}(Z)=Z^{n}+\sum_{j=1}^{n} a_{j} Z^{n-j}
$$

be a unitary polynomial with coefficients $a=\left(a_{1}, \ldots, a_{n}\right) \in \mathbb{C}^{n}$. We denote by $\xi(a)=$ $\left\{\xi_{1}(a), \ldots, \xi_{n}(a)\right\}$ the unordered set of roots of $P_{a}$ and assign to $a_{i}$ the weight $i$ so that homogeneous permutation invariant polynomials in $\xi$ are precisely the weighted homogeneous polynomials in $a$.

Let $N \in \mathbb{N}$ be a large constant fixed throughout the paper $\left(N \geq \max _{1 \leq s \leq n}\left(\begin{array}{l}n \\ s\end{array}\right)\right)$. For $2 \leq$ $m \leq n$ we denote by $\mathscr{D}_{N, m}$, or simply by $\mathscr{D}_{m}$, the ideal of $\mathbb{C}[a]$ generated by all $f^{N ! / s} \in \mathbb{C}[a]$, where $f$ runs over all homogeneous polynomials in $\prod_{i \neq j \in I}\left(\xi_{i}-\xi_{j}\right), I \subset\{1, \ldots, n\},|I|=m$, of degree $s \leq N$ in $\xi$, that are invariant by the permutations of $\xi_{i}$.

Example 1.1. $\mathscr{D}_{N, n}$ is the principal ideal generated by the $N ! / n(n-1)$-th power of the discriminant of $P_{a}$. 
By replacing $Z$ by $Z-a_{1} / n$ we define a new polynomial

$$
P_{\hat{a}}(Z)=Z^{n}+\hat{a}_{2} Z^{n-2}+\cdots+\hat{a}_{n}:=P_{a}\left(Z-a_{1} / n\right) .
$$

Each $\hat{a}_{j}$ is a weighted homogeneous polynomial in the $a_{i}$ 's of the weighted degree $j$. This transformation $a \rightarrow \hat{a}$, called Tschirnhausen transformation, shifts the roots of $P_{a}$ by $a_{1} / n$. Since the polynomials in $\prod_{i \neq j \in I}\left(\xi_{i}-\xi_{j}\right)$ are invariant by shifts of the roots, Tschirnhausen transformation does not change the ideal $\mathscr{D}_{m}$. Therefore, in what follows, we may suppose without loss of generality that $P_{a}$ is in Tschirnhausen form

$$
P_{a}(Z)=Z^{n}+a_{2} Z^{n-2}+\cdots+a_{n}
$$

Proposition 1.2. Suppose that $P_{a}$ is in Tschirnhausen form (1.2). Then $\mathscr{D}_{N, 2}$ is the ideal of $\mathbb{C}\left[a_{2}, \ldots, a_{n}\right]$ generated by the weighted homogeneous polynomials in $a_{2}, \ldots, a_{n}$ of weighted degree $N$ !.

Proof. If $P_{a}$ is in Tschirnhausen form then $\sum_{i=1}^{n} \xi_{i}=0$ and hence $\xi_{i}=\frac{1}{n} \sum_{j}\left(\xi_{i}-\xi_{j}\right)$. Therefore any polynomial in the $\xi_{i}$ 's is a polynomial in the $\left(\xi_{i}-\xi_{j}\right)$ 's.

Thus the zero set $V\left(\mathscr{D}_{2}\right)$ of $\mathscr{D}_{2}$ is exactly the set of those $a$ for which $P_{a}$ has precisely one root (i.e. $a_{2}=\cdots=a_{n}=0$ if $P_{a}$ is in Tschirnhausen form (1.2) and then this root is zero). In general, the zero set of $\mathscr{D}_{m}$ consists of those $a \in \mathbb{C}^{n}$ for which $P_{a}$ has at most $m-1$ distinct roots, cf. Corollary 6.4 below.

1.2. Smooth principalization of an ideal. Let $\mathscr{I}=\left(f_{1}, \ldots, f_{s}\right)$ be an ideal of $\mathbb{C}[x]$, $x=\left(x_{1}, \ldots, x_{m}\right)$. We shall assume $\mathscr{I} \neq(0)$. The blowing-up of $\mathscr{I}, \sigma: M=B l_{\mathscr{I}} \mathbb{C}^{m} \rightarrow \mathbb{C}^{m}$ can then be realized as follows, see for instance [10] Example 7.17.2. The variety $M$ is the irreducible component of

$$
\left\{(x, y) \in \mathbb{C}^{m} \times \mathbb{P}^{s-1} ; f_{i}(x) y_{j}=f_{j}(x) y_{i}, i, j=1, \ldots, s\right\}
$$

that projects surjectively onto $\mathbb{C}^{m}$ and $\sigma$ is the projection on the first factor. Then $M$ is the union of finitely many standard affine charts $\mathcal{V}_{i}$, where

$$
\mathcal{V}_{i}=M \cap\left\{\left(x, y_{1}, \ldots, \widehat{y}_{i} \ldots, y_{s}\right) \in \mathbb{C}^{m} \times \mathbb{C}^{s-1} ; f_{i}(x) y_{j}=f_{j}(x), j=1, \ldots, \widehat{i}, \ldots, s\right\}
$$

is a subvariety of the affine space $\mathbb{C}^{m} \times \mathbb{C}^{s-1}=\left\{(x, y) \in \mathbb{C}^{m} \times \mathbb{P}^{s-1} ; y_{i}=1\right\}$ with the coordinates $x_{j}, j=1, \ldots, m$, and $y_{j}=f_{j} / f_{i}, j=1, \ldots, \widehat{i}, \ldots, s$. The pullback of $\mathscr{I}$ on $\mathcal{V}_{i}$ is generated by $f_{i}$, since $f_{j}=y_{j} f_{i}$ for $j \neq i$, and hence is an invertible ideal, i.e. principal and generated by a non-zero divisor. The pullback of $\mathscr{I}$ on $M$, denoted by $\sigma^{*}(\mathscr{I})$, is an invertible sheaf of ideals.

In general, for arbitrary $\mathscr{I}$, the blow-up space $B l_{\mathscr{I}} \mathbb{C}^{m}$ is singular. Using Hironaka's resolution of singularities [11, it is possible to give an algorithm of principalization of any ideal by composing a sequence of smooth blow-ups (that is blowing-ups with smooth nowhere dense centers). Then the blow-up space is non-singular. Such a principalization map is not unique. We will use the following theorem that is a special case of Principalization III of [13], see also Theorem 1.10 of [3].

Theorem 1.3. Let $\mathscr{I} \neq(0)$ be an ideal of $\mathbb{C}[x]$. Then there exists a composition of smooth blowing-ups, $\sigma: M \rightarrow \mathbb{C}^{m}$, such that : 
(1) $\sigma^{*}(\mathscr{I})$ is invertible and its zero set $V\left(\sigma^{*}(\mathscr{I})\right)$ is a simple normal crossing divisor.

(2) The restriction $\left.\sigma\right|_{M \backslash V\left(\sigma^{*}(\mathscr{I})\right)}: M \backslash V\left(\sigma^{*}(\mathscr{I})\right) \rightarrow \mathbb{C}^{m} \backslash V(\mathscr{I})$ is an isomorphism.

Recall that a simple normal crossing divisor $E \subset M$ is the union $E=\cup_{i} E_{i}$ of nonsingular hypersurfaces $E_{i}$ intersecting transversally.

1.3. Privileged coordinate system. The map $\sigma$ of Theorem 1.3 is itself a blowing-up of an ideal $\mathscr{K} \subset \mathbb{C}[x]$ such that $V(\mathscr{K})=V(\mathscr{I})$. (Because $\sigma$ is birational and projective, see [10], Ch. II Thm. 7.17 and Exercise 7.11 (c)). Thus $M=B l_{\mathscr{K}} \mathbb{C}^{m}$. Denote $V\left(\sigma^{*}(\mathscr{I})\right)$ by $E$ and let $E=\cup_{i} E_{i}$ be the decomposition into irreducible components.

Let $\mathscr{I}=\left(f_{1}, \ldots, f_{s}\right)$ and $\mathscr{K}=\left(h_{1}, \ldots, h_{l}\right)$ and let $\mathcal{V} \subset B l_{\mathscr{K}} \mathbb{C}^{m}$ be a standard affine chart of $B l_{\mathscr{K}} \mathbb{C}^{m}$. Then on $\mathcal{V}, \sigma^{*}(\mathscr{I})$ is a principal ideal that is generated by some $f_{i}$, say $f_{1}$, and $\sigma^{*}(\mathscr{K})$ by some $h_{i}$, say $h_{1} \cdot \mathcal{V}$ is a subvariety of $\mathbb{C}^{m} \times \mathbb{C}^{l-1}$ with the coordinates $x_{i}, i=1, \ldots, m$, and $h_{j} / h_{1}, j=2, \ldots, l$. Each component $E_{i} \cap \mathcal{V}$ is the zero set of a finite number of functions regular on $\mathcal{V}$, that is functions of the form $P / h_{1}^{s}, s \in \mathbb{N}$, where $P$ is a polynomial in $x$.

Let $p \in \mathcal{V}$. There is a neighborhood $\mathcal{U}$ of $p$ in $\mathcal{V}$, and a coordinate system $y_{1}, \ldots, y_{m}$ on $\mathcal{U}$, such that $y_{i}=P_{i} / h_{1}^{s}, P_{i} \in \mathbb{C}[x], s \in \mathbb{N}$, and $E \cap \mathcal{U}$ is given by $y_{1} \cdots y_{r}=0$. (By taking the maximum we may choose $s$ independent of $i$.) Then

$$
f_{1}=\text { unit } \cdot \prod_{i=1}^{r} y_{i}^{n_{i}}, \quad h_{1}=\text { unit } \cdot \prod_{i=1}^{r} y_{i}^{m_{i}},
$$

with $n_{i}>0$ and $m_{i}>0$. Here by a unit we mean a function defined, analytic, and nowhere vanishing on $\mathcal{U}$.

1.4. Tower of smooth principalizations. For the ideals $\mathscr{D}_{m}, m=2, \ldots, n$, we construct a tower of smooth principalizations

$$
M_{1}=\mathbb{C}^{n} \stackrel{\sigma_{2}}{\longleftarrow} M_{2} \stackrel{\sigma_{3,2}}{\longleftarrow} M_{3} \stackrel{\sigma_{4,3}}{\longleftarrow} \ldots \stackrel{\sigma_{n, n-1}}{\longleftarrow} M_{n}
$$

We take as $\sigma_{2}$ a smooth principalization of $\mathscr{D}_{2}$ satisfying the conclusion of Theorem 1.3. Given $\sigma_{2}, \sigma_{3,2}, \ldots, \sigma_{m, m-1}$, we denote by $\sigma_{m}: M_{m} \rightarrow \mathbb{C}^{n}$ the composition $\sigma_{m}=\sigma_{2} \circ \sigma_{3,2} \circ \cdots \circ \sigma_{m, m-1}$, and take as $\sigma_{m+1, m}$ a smooth principalization of $\sigma_{m}^{*}\left(\mathscr{D}_{m+1}\right)$. Then $\sigma_{m+1}=\sigma_{m} \circ \sigma_{m+1, m}$ is a smooth principalization of $\mathscr{D}_{m+1}$. We denote $\sigma_{n, m}=\sigma_{m+1, m} \circ \cdots \circ \sigma_{n, n-1}$.

By Subsection 1.3, $\sigma_{m}$ is the blowing-up of an ideal $\mathscr{K}_{m} \subset \mathbb{C}[a]$.

Definition 1.4. By local data $\left(f, h, P_{i}, s, r\right)$ for $p \in M_{m}$ we mean the following. A polynomial $f \in \mathscr{D}_{m}$ that generates $\sigma_{m}^{*}\left(\mathscr{D}_{m}\right)$ at $p$, a polynomial $h \in \mathscr{K}_{m}$ that generates $\sigma_{m}^{*}\left(\mathscr{K}_{m}\right)$, a positive integer $s$ and polynomials $P_{i}$ such that $y_{i}=P_{i} / h^{s}, i=1, \ldots, n$, is a privileged system of coordinates in a neighborhood $\mathcal{U}$ of $p$, and $r$ such that $f^{-1}(0)$ is given by $y_{1} \cdots y_{r}=0$.

We fix such local data for every $p \in M_{m}$ (but allow to replace the neighborhood $\mathcal{U}$ by a smaller one if necessary).

Definition 1.5. By a chain $\mathrm{C}=\left(p_{m}, f_{m}, h_{m}, P_{m, i}, s_{m}, r_{m}\right)$ for $p_{n} \in M_{n}$ we mean the points $p_{m}:=\sigma_{n, m}\left(p_{n}\right), m=1, \ldots, n$, and the local data $\left(f_{m}, h_{m}, P_{m, i}, s_{m}, r_{m}\right)$ for $p_{m}$. We complete this data for $m=1$ by putting $f_{1}=h_{1}=1, P_{1, i}=a_{i}$, and $s_{1}=r_{1}=0$. 
When we specify the neighborhoods $\mathcal{U}_{m} \subset M_{m}$ of $p_{m}$ on which these local data are defined we always assume that $\sigma_{m, m-1}\left(\mathcal{U}_{m}\right) \subset \mathcal{U}_{m-1}$.

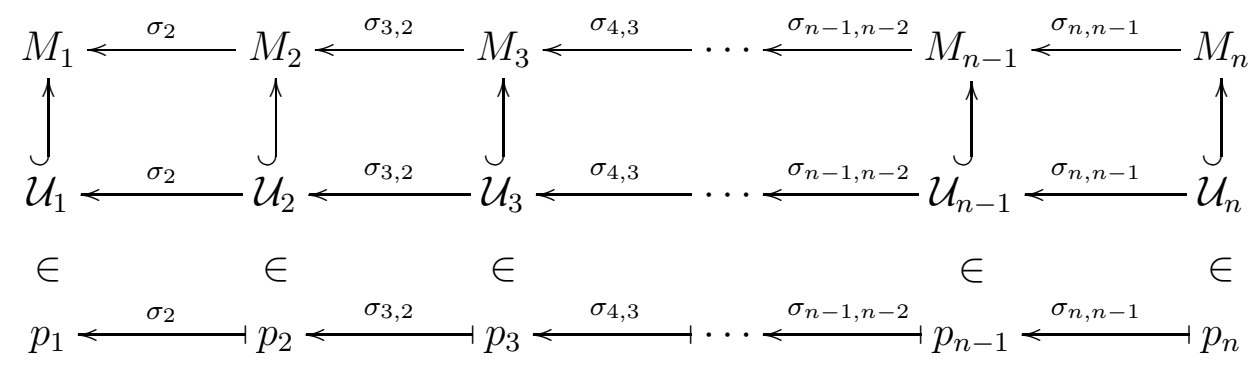

We pull back the polynomial $P_{a}$ onto $M_{m}$ via $\sigma_{m}$,

$$
P_{\sigma_{m}^{*}(a)}(Z)=Z^{n}+\sum_{i=1}^{n}\left(a_{i} \circ \sigma_{m}\right) Z^{n-i} .
$$

The roots of $P_{\sigma_{n}^{*}(a)}$ are the pull-backs of the roots of $P_{a}$.

\subsection{Formulas for the roots.}

Theorem 1.6. [Formulas for the roots]

Given a tower (1.4), we may associate with every $p_{m} \in M_{m}$ a convergent power series $\psi_{m}$, an integer $q_{m} \geq 1$, and a positive exponent $\alpha_{m} \in \frac{1}{q_{m}} \mathbb{N}_{>0}$, such that the following holds. For any chain $\mathrm{C}=\left(p_{m}, f_{m}, h_{m}, P_{m, i}, s_{m}, r_{m}\right)$ the roots of $P_{\sigma_{n}^{*}(a)}$ in a neighborhood of $p_{n}$ are given by

$$
\sum_{m=1}^{n} A_{m} \varphi_{m} \circ \sigma_{n, m}
$$

where $A_{m} \in \mathbb{Q}$ and

$$
\varphi_{m}=f_{m}^{\alpha_{m}} \psi_{m}\left(y_{m, 1}^{1 / q_{m}}, \ldots, y_{m, r_{m}}^{1 / q_{m}}, y_{m, r_{m}+1}, \ldots, y_{m, n}\right) .
$$

Theorem 1.6 will be proved in Section 9.

Remark 1.7. Because $f_{m}$ is a normal crossing in $y_{m, i}$, cf. (1.3),

$$
f_{m}^{\alpha_{m}} \in \mathbb{C}\left\{\left(y_{m, 1}^{1 / q_{m}}, \ldots, y_{m, r_{m}}^{1 / q_{m}}, y_{m, r_{m}+1}, \ldots, y_{m, n}\right)\right\} .
$$

Hence $\varphi_{m}$ of (1.6) is a fractional power series. It can be interpreted geometrically as follows. Set

$$
y_{m, i}= \begin{cases}t_{i}^{q_{m}} & \text { if } i \leq r_{m} \\ t_{i} & \text { if } i>r_{m}+1 .\end{cases}
$$

Then $\varphi_{m}$ is a convergent power series in $t=\left(t_{1}, \ldots, t_{n}\right)$. There are neighborhoods $\mathcal{U}_{m}$ of $p_{m}, \sigma_{m, m-1}\left(\mathcal{U}_{m}\right) \subset \mathcal{U}_{m-1}$, and their branched covers $\tau_{m}: \widetilde{\mathcal{U}}_{m} \rightarrow \mathcal{U}_{m}$, given by the formulas (1.7), such that $\psi_{m} \circ \tau_{m}$ and $\varphi_{m} \circ \tau_{m}$ are analytic on $\widetilde{\mathcal{U}}_{m}$. Since $\sigma_{m+1, m}^{-1}\left(f_{m}^{-1}(0)\right) \subset f_{m+1}^{-1}(0)$, $y_{m, i} \circ \sigma_{m+1, m}$, for $i \leq r_{m}$, is a normal crossings in $y_{m+1,1}, \ldots, y_{m+1, r_{m+1}}$ and therefore, we may 
suppose that $\sigma_{m+1, m} \circ \tau_{m+1}$ factors through $\tau_{m}$, changing $q_{m+1}$ if necessary. Thus we obtain a sequence of branched covers $\tau_{i}$ making the following diagram commutative.

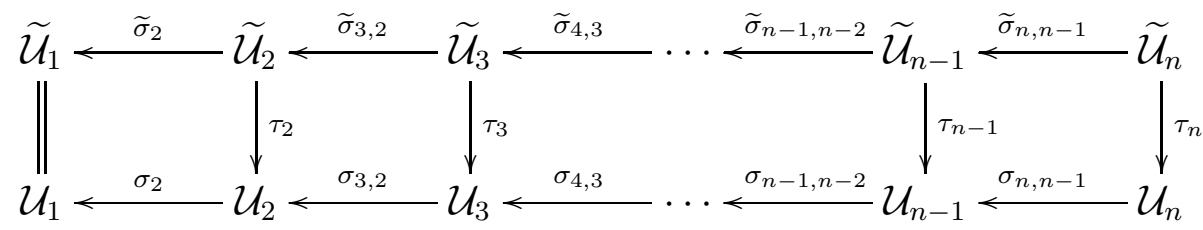

Then Theorem 1.6 says that the roots of $P_{\tilde{\sigma}_{n}^{*}(a)}$ are combinations of analytic functions on $\widetilde{\mathcal{U}}_{n}$ that are pull-backs of analytic functions on the $\widetilde{\mathcal{U}}_{m}$ 's.

Definition 1.8. By an extended chain $\mathrm{E}=\left(p_{m}, f_{m}, h_{m}, P_{m, i}, s_{m}, r_{m}, \mathcal{U}_{m}\right)$ for $p_{n} \in M_{n}$ we mean a chain $\mathrm{C}=\left(p_{m}, f_{m}, h_{m}, P_{m, i}, s_{m}, r_{m}\right)$ and a system of neighborhoods $\mathcal{U}_{m}$ of $p_{m}$ as in Remark 1.7. By Theorem [1.6 for every $p_{n} \in M_{n}$ there is an extended chain.

We filter the coefficient space $\mathbb{C}^{n}$ by the zero sets of discriminant ideals $\Sigma_{m}:=V\left(\mathscr{D}_{m}\right)$,

$$
\mathbb{C}^{n} \supset \Sigma_{n} \supset \cdots \supset \Sigma_{2}
$$

By Corollary 6.4, $a \in \Sigma_{m}$ if and only if $P_{a}(Z)$ has at most $m-1$ distinct roots. If $a(t)$ : $\mathbb{R} \supset I \rightarrow \mathbb{C}^{n}$ is continuous then $\Omega_{m}:=I \backslash a^{-1}\left(\Sigma_{m}\right)$ defines a filtration by open subsets $I \supset \Omega_{2} \supset \cdots \supset \Omega_{n}$. Because $\sigma_{m}$ is an isomorphism over $\mathbb{C}^{n} \backslash \Sigma_{m},\left.a\right|_{\Omega_{m}}$ has a lift $\widehat{a}_{m}$ to $M_{m}$. For $m=n$ we write $\widehat{a}:=\widehat{a}_{n}$.

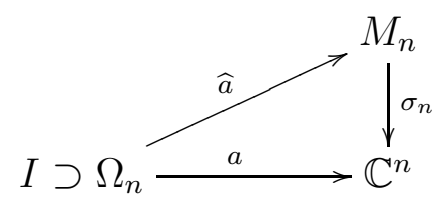

Lemma 1.9. [Addendum to Theorem 1.6] Let $\mathrm{E}=\left(p_{m}, f_{m}, h_{m}, P_{m, i}, s_{m}, r_{m}, \mathcal{U}_{m}\right)$ be an extended chain for $p_{n} \in M_{n}$ and let $J$ be $a$ connected component of $\widehat{a}^{-1}\left(\mathcal{U}_{n}\right)$. Let $\lambda(t)$ be a continuous root of $P_{a(t)}(Z)$ on $J$. Then there are continuous choices of radicals $f_{m}^{\alpha_{m}}(a(t))$ and $y_{m, 1}^{1 / q_{m}}\left(\widehat{a}_{m}(t)\right), \ldots, y_{m, r_{m}}^{1 / q_{m}}\left(\widehat{a}_{m}(t)\right)$, such that

$$
\lambda(t)=\sum_{m=1}^{n} A_{m} \varphi_{m}(t),
$$

where

$$
\varphi_{m}(t)=f_{m}^{\alpha_{m}}(a(t)) \psi_{m}\left(y_{m, 1}^{1 / q_{m}}\left(\widehat{a}_{m}(t)\right), \ldots, y_{m, r_{m}}^{1 / q_{m}}\left(\widehat{a}_{m}(t)\right), y_{m, r_{m}+1}\left(\widehat{a}_{m}(t)\right), \ldots, y_{m, n}\left(\widehat{a}_{m}(t)\right)\right) .
$$

Lemma 1.9 will be proved in Subsection 9.1.

\section{FunCtion SPACES}

In this section we fix notation for function spaces and present an extension lemma. 
2.1. Function spaces. Let $\Omega \subset \mathbb{R}^{n}$ be open and bounded. We denote by $C^{0}(\Omega)$ the space of continuous complex-valued functions on $\Omega$. For $k \in \mathbb{N} \cup\{\infty\}$ we set

$$
\begin{aligned}
& C^{k}(\Omega)=\left\{f \in \mathbb{C}^{\Omega} ; \partial^{\alpha} f \in C^{0}(\Omega), 0 \leq|\alpha| \leq k\right\}, \\
& C^{k}(\bar{\Omega})=\left\{f \in C^{k}(\Omega) ; \partial^{\alpha} f \text { has a continuous extension to } \bar{\Omega}, 0 \leq|\alpha| \leq k\right\},
\end{aligned}
$$

where $\bar{\Omega}$ denotes the closure of $\Omega$.

Note that $C^{k}(\bar{\Omega})$ is a Banach space when equipped with the norm

$$
\|f\|_{C^{k}(\bar{\Omega})}:=\sup _{\substack{|\alpha| \leq k \\ x \in \Omega}}\left|\partial^{\alpha} f(x)\right| .
$$

For $k \in \mathbb{N}$ and $p \geq 1$ we consider the Sobolev space

$$
W^{k, p}(\Omega)=\left\{f \in L^{p}(\Omega) ; \partial^{\alpha} f \in L^{p}(\Omega), 0 \leq|\alpha| \leq k\right\},
$$

where $\partial^{\alpha} f$ denote distributional derivatives. On bounded intervals $I \subset \mathbb{R}$ the Sobolev space $W^{1,1}(I)$ coincides with the space $A C(I)$ of absolutely continuous functions on $I$ if we identify each $W^{1,1}$-function with its unique continuous representative. Recall that a function $f: \Omega \rightarrow \mathbb{R}$ on an open subset $\Omega \subset \mathbb{R}$ is absolutely continuous if for every $\epsilon>0$ there exists $\delta>0$ so that $\sum_{i=1}^{n}\left|a_{i}-b_{i}\right|<\delta$ implies $\sum_{i=1}^{n}\left|f\left(a_{i}\right)-f\left(b_{i}\right)\right|<\epsilon$ whenever $\left[a_{i}, b_{i}\right], i=1, \ldots, n$, are non-overlapping intervals contained in $\Omega$.

Let $\Omega \subset \mathbb{R}^{n}$ be open and bounded, and let $1 \leq p<\infty$. A measurable function $f: \Omega \rightarrow \mathbb{C}$ belongs to the weak $L^{p}$-space $L_{w}^{p}(\Omega)$ if

$$
\|f\|_{p, w, \Omega}:=\sup _{r \geq 0}\left\{r \cdot \mathcal{L}^{n}(\{x \in \Omega ;|f(x)|>r\})^{\frac{1}{p}}\right\}<\infty
$$

where $\mathcal{L}^{n}$ denotes the $n$-dimensional Lebesgue measure. For $1 \leq q<p<\infty$ we have (cf. [9] Example 1.1.11)

$$
\|f\|_{q, w, \Omega} \leq\|f\|_{L^{q}(\Omega)} \leq\left(\frac{p}{p-q}\right)^{\frac{1}{q}} \mathcal{L}^{n}(\Omega)^{\frac{1}{q}-\frac{1}{p}}\|f\|_{p, w, \Omega}
$$

and hence $L^{p}(\Omega) \subset L_{w}^{p}(\Omega) \subset L^{q}(\Omega) \subset L_{w}^{q}(\Omega)$ with strict inclusions. Note that \|\|$_{p, w, \Omega}$ is only a quasinorm; more precisely, for $f_{j} \in L_{w}^{p}(\Omega)$ we have

$$
\left\|\sum_{j=1}^{m} f_{j}\right\|_{p, w, \Omega} \leq m \sum_{j=1}^{m}\left\|f_{j}\right\|_{p, w, \Omega} .
$$

If $\Omega_{i}$ is a finite or countable family of open sets whose union is $\Omega$ then (cf. [7] Lemma 3.1)

$$
\|f\|_{p, w, \Omega} \leq \sum_{i}\|f\|_{p, w, \Omega_{i}}, \quad \forall f \in L_{w}^{p}(\Omega)
$$

If $p>1$ then there exists a norm equivalent to \|\|$_{p, w, \Omega}$ which makes $L_{w}^{p}(\Omega)$ into a Banach space.

Analogously we may consider $L_{w}^{p}(K)$ for compact sets $K \subset \mathbb{R}^{n}$. We shall also use $W_{\text {loc }}^{k, p}$, $A C_{\text {loc }}$, as well as $L^{p}\left(\Omega, \mathbb{C}^{m}\right)=\left[L^{p}(\Omega)\right]^{m}, W^{k, p}\left(\Omega, \mathbb{C}^{m}\right)=\left[W^{k, p}(\Omega)\right]^{m}$, etc., with the obvious meaning. 
2.2. Extension lemma. The following lemma is a generalization of Lemma 3.2 of [7] with essentially the same proof.

Lemma 2.1. Let $\Omega \subset \mathbb{R}$ be open and bounded, let $f: \Omega \rightarrow \mathbb{C}$ be continuous, and set $\Omega_{0}:=\{t \in \Omega ; f(t) \neq 0\}$. Assume that $\left.f\right|_{\Omega_{0}} \in A C_{\mathrm{loc}}\left(\Omega_{0}\right)$ and that $\left.f\right|_{\Omega_{0}} ^{\prime} \in L_{w}^{p}\left(\Omega_{0}\right)$ for some $p>1$ (note that $f$ is differentiable a.e. in $\Omega_{0}$ ). Then the distributional derivative of $f$ in $\Omega$ is a measurable function $f^{\prime} \in L_{w}^{p}(\Omega)$ and

$$
\left\|f^{\prime}\right\|_{p, w, \Omega}=\left\|\left.f\right|_{\Omega_{0}} ^{\prime}\right\|_{p, w, \Omega_{0}} .
$$

Proof. The function $\psi: \Omega \rightarrow \mathbb{C}$ defined by

$$
\psi(t):= \begin{cases}f^{\prime}(t) & \text { if } t \in \Omega_{0} \\ 0 & \text { if } t \in \Omega \backslash \Omega_{0}\end{cases}
$$

clearly belongs to $L_{w}^{p}(\Omega)$. We show that $\psi$ is the distributional derivative of $f$ in $\Omega$. Let $\phi \in C_{c}^{\infty}(\Omega)$ be a test function with compact support in $\Omega$ and let $\mathcal{C}$ denote the (at most countable) set of connected components of $\Omega_{0}$. Then, using integration by parts for the Lebesgue integral (see e.g. [15] Corollary 3.37)

$$
\int_{\Omega} f \phi^{\prime} d t=\int_{\Omega_{0}} f \phi^{\prime} d t=\sum_{J \in \mathcal{C}} \int_{J} f \phi^{\prime} d t=-\sum_{J \in \mathcal{C}} \int_{J} f^{\prime} \phi d t=-\int_{\Omega_{0}} f^{\prime} \phi d t=-\int_{\Omega} \psi \phi d t .
$$

(If $J=(a, b)$ then $\int_{a}^{b} f \phi^{\prime} d t=\lim _{\epsilon \rightarrow 0^{+}} \int_{a+\epsilon}^{b-\epsilon} f \phi^{\prime} d t=-\lim _{\epsilon \rightarrow 0^{+}} \int_{a+\epsilon}^{b-\epsilon} f^{\prime} \phi d t=-\int_{a}^{b} f^{\prime} \phi d t$, by the Dominated Convergence Theorem, continuity of $f$, and (2.1).) Moreover, we have $\left\|f^{\prime}\right\|_{p, w, \Omega}=\|\psi\|_{p, w, \Omega}=\|\psi\|_{p, w, \Omega_{0}}=\left\|\left.f\right|_{\Omega_{0}} ^{\prime}\right\|_{p, w, \Omega_{0}}$.

\section{Absolute continuity of roots}

3.1. Optimal regularity of radicals of differentiable functions. We need the following variant of Theorem 2.2 of [7].

Proposition 3.1. Let $I \subset \mathbb{R}$ be a bounded interval and let $k \in \mathbb{N}_{>0}$. For each $f \in C^{k}(\bar{I}, \mathbb{C})$ we have

$$
\left|f^{\prime}(t)\right| \leq \Lambda_{k}(t)|f(t)|^{1-\frac{1}{k}} \quad \text { a.e. in } I
$$

for some $\Lambda_{k}=\Lambda_{k, f} \in L_{w}^{p}\left(I, \mathbb{R}_{\geq 0}\right)$, where $\frac{1}{p}+\frac{1}{k}=1$, and such that

$$
\left\|\Lambda_{k}\right\|_{p, w, I} \leq C(k) \max \left\{\left\|f^{(k)}\right\|_{L^{\infty}(I)}^{\frac{1}{k}}|I|^{\frac{1}{p}},\left\|f^{\prime}\right\|_{L^{\infty}(I)}^{\frac{1}{k}}\right\} .
$$

Proof. If the real and imaginary part of $f$ satisfy (3.1), then so does $f$. Hence it suffices to consider the case that $f$ is real-valued.

Set $h=|f|^{\frac{1}{k}}$. Then Theorem 2.2 of [7] implies that $h^{\prime} \in L_{w}^{p}(I, \mathbb{R})$ and

$$
\left\|h^{\prime}\right\|_{p, w, I} \leq C(k) \max \left\{\left\|f^{(k)}\right\|_{L^{\infty}(I)}^{\frac{1}{k}}|I|^{\frac{1}{p}},\left\|f^{\prime}\right\|_{L^{\infty}(I)}^{\frac{1}{k}}\right\} .
$$

In particular, $h \in W^{1, q}(I, \mathbb{R})$ for each $q \in[1, p)$. By differentiating $h^{k}=|f|$ we find

$$
\left|f^{\prime}(t)\right|=k\left|h^{\prime}(t)\right||f(t)|^{1-\frac{1}{k}} \quad \text { for all } t \in I \text { with } f(t) \neq 0 \text {. }
$$


The derivative $f^{\prime}$ vanishes at the accumulation points of $f^{-1}(0)$, and the isolated points of $f^{-1}(0)$ form an at most countable set. So we conclude that (3.1) holds with $\Lambda_{k}:=k\left|h^{\prime}\right|$.

Remark 3.2. Proposition 3.1 is optimal in the following sense. $\Lambda_{k}$ cannot, in general, be chosen in $L^{p}$. Indeed, for $f:(-1,1) \rightarrow \mathbb{R}, f(t)=t$, we have

$$
\left(\frac{\left|f^{\prime}\right|}{|f|^{1-\frac{1}{k}}}\right)^{p}=\left(|t|^{\frac{1}{k}-1}\right)^{p}=|t|^{-1}
$$

which is not integrable near 0. See [7] Example 4.3.

Remark 3.3. In Proposition 3.1 it is actually enough to require that $f \in C^{k-1,1}(\bar{I}, \mathbb{C})$; cf. [7] Theorem 2.2.

3.2. Set-valued functions and curves of polynomials. In the following we shall be dealing with multi-valued functions arising from complex radicals, their composition with single-valued functions, and their addition and multiplication.

The (usual) composition $G \circ F: X \leadsto Z$ of two set-valued functions $F: X \leadsto Y$ and $G: Y \leadsto Z$ is given by $(G \circ F)(x)=\cup_{y \in F(x)} G(y)$. The addition $F+G$ and multiplication $F G$ of $F: X \leadsto \mathbb{C}$ and $G: X \leadsto \mathbb{C}$ are then well-defined.

A selection of a set-valued function $F: X \leadsto Y$ is a single-valued function $f: X \rightarrow Y$ satisfying $f(x) \in F(x)$ for all $x \in X$. A parameterization of a set-valued function $F$ : $X \leadsto Y$ is a pair $(f, Z)$, where $f: X \times Z \rightarrow Y$ is a single-valued function so that $F(x)=$ $\{f(x, z) ; z \in Z\}$ for every $x \in X$. We shall only be concerned with multi-valued functions $F$ so that the cardinality $|F(x)|$ is finite and bounded, i.e., $\max _{x \in X}|F(x)|=: N<\infty$. Then a parameterization of $F$ is an $N$-tuple of single-valued functions (with multiplicities at points $x$ where $|F(x)|<N)$.

If the coefficients of the polynomial $P_{a}$ in (1.1) are complex-valued continuous functions $a_{j} \in C^{0}(I)$ defined in an interval $I \subset \mathbb{R}$, we say that $P_{a}(t)=P_{a(t)}, t \in I$, is a curve of polynomials. The roots of a curve of polynomials form a multi-valued function $\lambda: I \leadsto \mathbb{C}$ which admits a continuous parameterization; see [12] Chapter II Theorem 5.2. (This is no longer true if the parameter space is higher-dimensional due to monodromy.) Moreover, any continuous selection of $\lambda: I \leadsto \mathbb{C}$ can be completed to a continuous parameterization $\lambda_{1}, \ldots, \lambda_{n}$ such that $P_{a(t)}(Z)=\prod_{i}\left(Z-\lambda_{i}(t)\right)$; see [24] Lemma 6.17.

Lemma 3.4. Let $\alpha \in \mathbb{Q}_{>0}, q, s \in \mathbb{N}_{>0}$, and suppose that $k \geq\left\lceil\max \left\{\frac{s}{\alpha}, q\right\}\right\rceil, p=\frac{k}{k-1}$. Let $I \subset \mathbb{R}$ be a bounded interval and let $\mathcal{U} \subset \mathbb{C}^{n}$ be open and bounded. Let $\psi \in C^{1}(\overline{\mathcal{U}})$, $h, P_{j} \in C^{k}(\bar{I})$, and let $\Omega \subset I$ be an open subset of $I$ so that $y^{1 / q}(\Omega) \subset \mathcal{U}$, where we put $y=\left(y_{1}, \ldots, y_{n}\right)=\left(P_{1} / h^{s}, \ldots, P_{n} / h^{s}\right)$ and $y^{1 / q}=\left(y_{1}^{1 / q}, \ldots, y_{n}^{1 / q}\right)$. Consider the multi-valued function

$$
\varphi=h^{\alpha} \psi\left(y^{\frac{1}{q}}\right)=h^{\alpha} \psi\left(y_{1}^{\frac{1}{q}}, \ldots, y_{n}^{\frac{1}{q}}\right) .
$$

Then $\varphi$ admits a continuous parameterization on $\Omega$ and for any such parameterization $\phi$ the distributional derivative of $\phi$ in $\Omega$ is a measurable function $\phi^{\prime} \in L_{w}^{p}(\Omega)$ and

$$
\left\|\phi^{\prime}\right\|_{p, w, \Omega} \leq C_{1}(\alpha, s, q, \mathcal{U})\|\psi\|_{C^{1}(\overline{\mathcal{U}})} N_{\alpha, k, s, I}(h) \max _{j}\left\{H_{k, I}(h), H_{k, I}\left(P_{j}\right)\right\}
$$


for a positive constant $C_{1}(\alpha, s, q, \mathcal{U})$, where for any function $g \in C^{k}(\bar{I})$ we set

$$
N_{\alpha, k, s, I}(g):=\max \left\{\|g\|_{L^{\infty}(I)}^{\alpha-\frac{s}{k}},\|g\|_{L^{\infty}(I)}^{\alpha-\frac{1}{k}}\right\}, \quad H_{k, I}(g):=\max \left\{\left\|g^{(k)}\right\|_{L^{\infty}(I)}^{\frac{1}{k}}|I|^{\frac{1}{p}},\left\|g^{\prime}\right\|_{L^{\infty}(I)}^{\frac{1}{k}}\right\} .
$$

Proof. First we show that $\varphi$ admits a continuous parameterization on $\Omega$. Consider the open subsets $\Omega_{1} \subset \Omega_{0} \subset \Omega \subset I$ given by

$$
\Omega_{0}=\{t \in \Omega ; h(t) \neq 0\} \quad \text { and } \quad \Omega_{1}=\left\{t \in \Omega_{0} ; \forall j P_{j} \neq 0\right\} .
$$

Each multi-valued function $y_{j}^{1 / q}=\left(P_{j} / h^{s}\right)^{1 / q}$ has a continuous parameterization on $\Omega_{0}$ and thus so does $\psi\left(y^{1 / q}\right)$. The multi-valued function $h^{\alpha}$ has a continuous parameterization on $I$, which vanishes on the zero set of $h$. Since $\psi\left(y^{1 / q}\right)$ is bounded on $\Omega$, we may conclude that $\varphi$ admits a continuous parameterization on $\Omega$.

Let $\phi$ be any continuous parameterization of $\varphi$ on $\Omega$. Abusing notation we denote by $\phi$ also any single component in the parameterization $\phi$. Then $\phi$ is $C^{1}$ on $\Omega_{1}$ and its derivative satisfies

$$
\begin{aligned}
\left|\phi^{\prime}\right| \leq & \alpha\left|\psi\left(y^{\frac{1}{q}}\right)\right| \frac{\left|h^{\prime}\right|}{|h|^{1-\alpha}}+\frac{1}{q} \sum_{j=1}^{n}\left|\partial_{j} \psi\left(y^{\frac{1}{q}}\right)\right|\left(\frac{\left|P_{j}^{\prime}\right|}{\left|P_{j}\right|^{1-\frac{1}{q}}}|h|^{\alpha-\frac{s}{q}}+s\left|\frac{P_{j}}{h^{s}}\right|^{\frac{1}{q}} \frac{\left|h^{\prime}\right|}{|h|^{1-\alpha}}\right) \\
\leq & \alpha\left|\psi\left(y^{\frac{1}{q}}\right)\right| \frac{\left|h^{\prime}\right|}{|h|^{1-\frac{1}{k}}}|h|^{\alpha-\frac{1}{k}} \\
& +\frac{1}{q} \sum_{j=1}^{n}\left|\partial_{j} \psi\left(y^{\frac{1}{q}}\right)\right|\left(\frac{\left|P_{j}^{\prime}\right|}{\left|P_{j}\right|^{1-\frac{1}{k}}}\left|\frac{P_{j}}{h^{s}}\right|^{\frac{1}{q}-\frac{1}{k}}\left|h^{s}\right|^{\frac{\alpha}{s}-\frac{1}{k}}+s\left|\frac{P_{j}}{h^{s}}\right|^{\frac{1}{q}} \frac{\left|h^{\prime}\right|}{|h|^{1-\frac{1}{k}}}|h|^{\alpha-\frac{1}{k}}\right)
\end{aligned}
$$

Next we claim that $\phi$ is locally absolutely continuous on $\Omega_{0}$. Indeed, every continuous parameterization of $h^{\alpha}$, respectively $P_{j}^{1 / q}$, is $A C$ on $I$ by Proposition 3.1, and consequently every continuous parameterization of $y_{j}^{1 / q}=\left(P_{j} / h^{s}\right)^{1 / q}$ is $A C_{\text {loc }}$ on $\Omega_{0}$; note that on each compact subinterval of $\Omega_{0}$ any continuous parameterization of $1 / h^{s / q}$ is $C^{1}$. Since $\psi$ is $C^{1}$, we may infer from Lemma 2.1 of [17] that each continuous parameterization of $\psi\left(y^{1 / q}\right)$, and thus of $h^{\alpha} \psi\left(y^{1 / q}\right)$, is locally absolutely continuous on $\Omega_{0}$. This shows the claim.

In particular, $\phi$ is differentiable almost everywhere in $\Omega_{0}$. We argue that (3.4) holds almost everywhere in $\Omega_{0}$, if we define

$$
\frac{\left|P_{j}^{\prime}\right|}{\left|P_{j}\right|^{1-\frac{1}{k}}}:=0 \quad \text { on accumulation points of } P_{j}^{-1}(0) \text {. }
$$

Indeed, by Lemma 2.1 of [17] the chain rule holds almost everywhere and the derivative of any continuous, and hence absolutely continuous, parameterization of $P_{j}^{1 / q}$ exists almost everywhere and vanishes on accumulation points of $P_{j}^{-1}(0)$. The isolated points of $P_{j}^{-1}(0)$ form an at most countable set.

Applying Proposition 3.1 we may conclude that

$$
\left|\phi^{\prime}\right| \leq \Lambda_{k} \Psi \quad \text { a.e. in } \Omega_{0} \text { for some } \Psi \in L^{\infty}(\Omega, \mathbb{R}) \text { and } \Lambda_{k} \in L_{w}^{p}(I, \mathbb{R}) \text {. }
$$

Here we set $\Lambda_{k}=\max \left\{\Lambda_{k, h}, \Lambda_{k, P_{j}}\right\}$. 
Extending $\Psi$ by 1 on $I \backslash \Omega$ and using $L_{w}^{p} \cdot L^{\infty} \subset L_{w}^{p}$ we obtain

$$
\left|\phi^{\prime}\right| \leq \tilde{\Lambda}_{k} \quad \text { a.e. in } \Omega_{0} \text { for some } \tilde{\Lambda}_{k} \in L_{w}^{p}(I, \mathbb{R}) .
$$

Using Lemma 2.1 we may conclude that the distributional derivative of $\phi$ in $\Omega$ is a measurable function $\phi^{\prime} \in L_{w}^{p}(\Omega)$.

The estimate (3.3) follows from (3.5) $)$ (3.2), and (2.4).

\subsection{Main Theorem.}

Theorem 3.5. For every $n \in \mathbb{N}_{>0}$ there is $k=k(n) \in \mathbb{N}_{>0}$ and $p=p(n)>1$ such that the following holds. Let $I \subset \mathbb{R}$ be a compact interval and let

$$
P_{a(t)}(Z)=Z^{n}+\sum_{j=1}^{n} a_{j}(t) Z^{n-j} \in C^{k}(I)[Z]
$$

be a monic polynomial with coefficients $a_{j} \in C^{k}(I), j=1, \ldots, n$.

(1) Let $\lambda_{j} \in C^{0}(I), j=1, \ldots, n$, be a continuous parameterization of the roots of $P_{a}$ on $I$. Then the distributional derivative of each $\lambda_{j}$ in $I$ is a measurable function $\lambda_{j}^{\prime} \in L^{q}(I)$ for every $q \in[1, p)$. In particular, each $\lambda_{j} \in W^{1, q}(I)$ for every $q \in[1, p)$.

(2) This regularity of the roots is uniform. Let $\left\{P_{a_{\nu}} ; \nu \in \mathcal{N}\right\}$,

$$
P_{a_{\nu}(t)}(Z)=Z^{n}+\sum_{j=1}^{n} a_{\nu, j}(t) Z^{n-j} \in C^{k}(I)[Z], \nu \in \mathcal{N},
$$

be a family of curves of polynomials, indexed by $\nu$ in some set $\mathcal{N}$, so that the set of coefficients $\left\{a_{\nu, j} ; \nu \in \mathcal{N}, j=1, \ldots, n\right\}$ is bounded in $C^{k}(I)$. Then the set

$$
\left\{\lambda_{\nu} \in C^{0}(I) ; P_{a_{\nu}}\left(\lambda_{\nu}\right)=0 \text { on } I, \nu \in \mathcal{N}\right\}
$$

is bounded in $W^{1, q}(I)$ for every $q \in[1, p)$.

The rest of this section will be devoted to the proof of Theorem 3.5,

3.4. Definition of $k(n)$ and $p(n)$. Let $\mathrm{E}=\left(p_{m}, f_{m}, h_{m}, P_{m, i}, s_{m}, r_{m}, \mathcal{U}_{m}\right)$ be an extended chain. By (1.3), we may express $\varphi_{m}$ of (1.6) as follows

$$
\varphi_{m}=h_{m}^{\widetilde{\alpha}_{m}} \tilde{\psi}_{m}\left(y_{m, 1}^{1 / \tilde{q}_{m}}, \ldots, y_{m, r_{m}}^{1 / \tilde{q}_{m}}, y_{m, r_{m}+1}, \ldots, y_{m, n}\right),
$$

where $\widetilde{\alpha}_{k} \in \frac{1}{\tilde{q}_{m}} \mathbb{N}_{>0}$ and $\tilde{q}_{m}$ is a positive integer possibly much bigger than $q_{m}$. Then we define

$$
k_{\mathrm{E}}:=\max _{m}\left\lceil\max \left\{\frac{s_{m}}{\widetilde{\alpha}_{m}}, \tilde{q}_{m}\right\}\right\rceil,
$$

We fix an open bounded neighborhood $\mathcal{B}$ of the origin in $\mathbb{C}^{n}$ and a finite family of extended chains

$$
\mathcal{C V}=\left\{\mathrm{E}_{j}\right\}=\left\{\left(p_{j, m}, f_{j, m}, h_{j, m}, P_{j, m, i}, s_{j, m}, r_{j, m}, \mathcal{U}_{j, m}\right)\right\}
$$

such that

$$
\sigma_{n}^{-1}(\overline{\mathcal{B}}) \subset \bigcup_{j} \mathcal{U}_{j, n}
$$


Then we set

$$
k=k(n):=\max _{j} k_{\mathrm{E}_{j}}, \quad \text { and } \quad p=p(n):=\frac{k}{k-1} \in \mathbb{Q}_{>1} \cup\{\infty\} .
$$

3.5. Real analytic case. We begin the proof of Theorem 3.5 with the following special case. We suppose that $a(t)$ is real analytic and that $a(t) \in \mathcal{B}$ for all $t \in I$. We suppose moreover that the discriminant of $P_{a(t)}$ is not identically equal to zero. Under these assumptions we show that

$$
\left\|\lambda_{j}^{\prime}\right\|_{p, w, I} \leq C(\mathcal{C V}) \sum_{j, m} N_{\widetilde{\alpha}_{j, m}, k, s_{j, m}, I}\left(h_{j, m}(t)\right) \max _{i}\left\{H_{k, I}\left(h_{j, m}(t)\right), H_{k, I}\left(P_{j, m, i}(t)\right)\right\},
$$

where the constant $C(\mathcal{C V})$ depends only on the family $\mathcal{C V}$.

Recall that $\widehat{a}$ denotes the lift of $\left.a\right|_{\Omega_{n}}$ over $\sigma_{n}$, cf. Subsection 1.5. We remark that actually $a$ has a unique real analytic lift to $M_{n}$ on the whole interval $I$, by the universal property of blowing-ups, see [10] Proposition 7.14; but we will not use this fact.

All the roots of $P_{a(t)}$ on $\Omega_{n}$ are distinct and hence, by the Implicit Function Theorem, depend analytically on $t$. Thus Lemmas 3.4 and 1.9 give (3.8) with $I$ replaced by $\widehat{a}^{-1}\left(\mathcal{U}_{n}\right) \cap \Omega_{n}$. We set $I_{i}:=\widehat{a}^{-1}\left(\mathcal{U}_{i, n}\right)$ and $\Omega_{i, n}:=I_{i} \cap \Omega_{n}$. Then, by (2.3),

$$
\left\|\lambda_{j}^{\prime}\right\|_{p, w, \Omega_{n}} \leq \sum_{i}\left\|\lambda_{j}^{\prime}\right\|_{p, w, \Omega_{i, n}}
$$

Since $a(t)$ is real analytic, $a^{-1}\left(\Sigma_{n}\right)=I \backslash \Omega_{n}$ is finite, and hence the derivative $\lambda_{j}^{\prime}$ of $\lambda_{j}$ exists almost everywhere in $I$ and belongs to $L^{1}(I)$ by (2.1). It coincides with the distributional derivative of $\lambda_{j}$ in $I$, and $\left\|\lambda_{j}^{\prime}\right\|_{p, w, I}=\left\|\lambda_{j}^{\prime}\right\|_{p, w, \Omega_{n}}$. (If $\phi \in C_{c}^{\infty}(I)$ and $(a, b)$ is a connected component of $\Omega_{n}$, then $\int_{a}^{b} \lambda_{j} \phi^{\prime} d t=\lim _{\epsilon \rightarrow 0^{+}} \int_{a+\epsilon}^{b-\epsilon} \lambda_{j} \phi^{\prime} d t=\lambda_{j}(b) \phi(b)-\lambda_{j}(a) \phi(a)-\lim _{\epsilon \rightarrow 0^{+}} \int_{a+\epsilon}^{b-\epsilon} \lambda_{j}^{\prime} \phi d t=$ $\lambda_{j}(b) \phi(b)-\lambda_{j}(a) \phi(a)-\int_{a}^{b} \lambda_{j}^{\prime} \phi d t$, by the Dominated Convergence Theorem. Since $I \backslash \Omega_{n}$ is finite, all boundary terms cancel and thus $\int_{I} \lambda_{j} \phi^{\prime} d t=-\int_{I} \lambda_{j}^{\prime} \phi d t$.) This implies (3.8).

3.6. Weighted homogeneity. Let $a(t)$ be real analytic and suppose that the discriminant of $P_{a(t)}$ is not identically equal to zero. We do not assume any longer that $a(t) \in \mathcal{B}$ for all $t \in I$. We extend the bound of the previous subsection to such curves using the weighted homogeneity.

For $\eta>0$ and $a \in \mathbb{C}^{n}$ we define $\eta * a \in \mathbb{C}^{n}$ by $(\eta * a)_{i}=\eta^{i} a_{i}$. Then $\lambda$ is a root of $P_{a}$ if and only if $\eta \lambda$ is a root of $P_{\eta * a}$.

Fix $\rho \geq \max \left\{1, \sup _{t \in I}\|a(t)\|\right\}$. Then $\left\|\rho^{-1} * a\right\| \leq 1$. For a polynomial $g \in \mathbb{C}[a]$, set $\tilde{g}(t):=g\left(\rho^{-1} * a(t)\right)$. Then by (3.8)

$$
\left\|\lambda_{j}^{\prime}\right\|_{p, w, I} \leq \rho C(\mathcal{C V}) \sum_{j, m} N_{\widetilde{\alpha}_{j, m}, k, s_{j, m}, I}\left(\tilde{h}_{j, m}\right) \max _{i}\left\{H_{k, I}\left(\tilde{h}_{j, m}\right), H_{k, I}\left(\tilde{P}_{j, m, i}\right)\right\}
$$

3.7. General Case. Let $a \in C^{k}\left(I, \mathbb{C}^{n}\right)$. By the classical Weierstrass Theorem there is a sequence of polynomial curves $\left(a_{\nu}\right) \subset C^{\omega}\left(I, \mathbb{C}^{n}\right)$, such that

$$
a_{\nu} \longrightarrow a \quad \text { in } C^{k}\left(I, \mathbb{C}^{n}\right) \quad(\nu \rightarrow \infty)
$$

By replacing $a_{\nu}$ by $a_{\nu}+\left(0, \ldots, 0, \varepsilon_{\nu}\right)$, with $\varepsilon_{\nu}>0$ sufficiently small, we may suppose moreover that the discriminant of each $P_{a_{\nu}}$ is not identically zero. 
For each $\nu$ choose a continuous parameterization $\lambda_{\nu}=\left(\lambda_{\nu, 1}, \ldots, \lambda_{\nu, n}\right) \in C^{0}\left(I, \mathbb{C}^{n}\right)$ of the roots of $P_{a_{\nu}}(t), t \in I$. Since $\left(a_{\nu}\right)$ is bounded in $C^{k}\left(I, \mathbb{C}^{n}\right)$, we may infer from (3.8) that the set of distributional derivatives $\left\{\lambda_{\nu}^{\prime} ; \nu\right\}$ is bounded in $L^{q}\left(I, \mathbb{C}^{n}\right)$ for every $q \in[1, p)$.

Fix $q \in(1, p)$. By the Arzelá-Ascoli Theorem, as $\left(\lambda_{\nu}\right)$ is equi-Hölder, or alternatively by the Rellich-Kondrachov Compactness Theorem, there is a subsequence $\left(\lambda_{\nu(\ell)}\right)$ that converges in $C^{0}\left(I, \mathbb{C}^{n}\right)$ to some $\lambda$.

Since $L^{q}(I)$ is reflexive, we also have (after possibly passing to a subsequence again) that $\left(\lambda_{\nu(\ell)}^{\prime}\right)$ converges to some $\lambda^{\prime}$ weakly in $L^{q}\left(I, \mathbb{C}^{n}\right)$. Then $\lambda^{\prime}$ is the distributional derivative of $\lambda$ and thus $\lambda \in W^{1, q}\left(I, \mathbb{C}^{n}\right)$. It is clear that $\lambda$ forms a parameterization of the roots of $P_{a}$ on $I$.

Lemma 3.6. Let $\lambda=\left(\lambda_{1}, \ldots, \lambda_{n}\right)$ and $\mu=\left(\mu_{1}, \ldots, \mu_{n}\right)$ be two parameterizations of the roots of $P_{a}(t)(Z) \in C^{k}(I)[Z]$. If $\lambda \in W^{1, q}\left(I, \mathbb{C}^{n}\right), q \in[1, p)$, and $\mu \in C^{0}\left(I, \mathbb{C}^{n}\right)$, then also $\mu \in W^{1, q}\left(I, \mathbb{C}^{n}\right)$ and

$$
\sum_{i=1}^{n}\left\|\mu_{i}^{\prime}\right\|_{L^{q}(I)}^{q}=\sum_{i=1}^{n}\left\|\lambda_{i}^{\prime}\right\|_{L^{q}(I)}^{q} .
$$

Proof. For each $j$ we have

$$
\operatorname{length}\left(\mu_{j}\right) \leq \sum_{i=1}^{n} \operatorname{length}\left(\lambda_{i}\right)<\infty
$$

and so $\mu_{j}$ is of bounded variation. Moreover, for any subset $E \subset I$

$$
\mu_{j}(E) \subset \bigcup_{i=1}^{n} \lambda_{i}(E)
$$

and hence $\mu_{j}$ has the Luzin $(\mathrm{N})$ property. We may conclude that each $\mu_{j}$ is absolutely continuous on $I$ and hence the derivative $\mu_{j}^{\prime}$ of $\mu_{j}$ exists almost everywhere in $I$ and coincides with the distributional derivative of $\mu_{j}$ in $I$.

At points $t$, where each $\mu_{j}$ and each $\lambda_{i}$ is differentiable, the sets $\left\{\mu_{j}^{\prime}(t)\right\}$ and $\left\{\lambda_{i}^{\prime}(t)\right\}$ coincide together with the multiplicities of its elements. These points form a subset of $I$ of full measure and therefore $\mu_{j}^{\prime} \in L^{q}(I)$ and satisfies (3.10).

Uniformity can be seen by repeating the proof with an additional parameter $\nu$. The weak limits in the reasoning above are weakly bounded and thus bounded in $L^{q}(I)$. 


\section{Multiparameter families of polynomials}

Theorem 4.1. Let $k=k(n) \in \mathbb{N}_{>0}$ and $p=p(n)>1$ be as in Subsection 3.4. Let $U \subset \mathbb{R}^{m}$ be open and let

$$
P_{a(x)}(Z)=Z^{n}+\sum_{j=1}^{n} a_{j}(x) Z^{n-j} \in C^{k}(U)[Z]
$$

be a monic polynomial with coefficients $a_{j} \in C^{k}(U), j=1, \ldots, n$.

(1) Let $\lambda \in C^{0}(V)$ represent a root of $P_{a}$, i.e., $P_{a}(\lambda)=0$, on a relatively compact open subset $V \Subset U$. Then the distributional gradient of $\lambda$ in $V$ is a measurable function $\nabla \lambda \in\left[L^{q}(V)\right]^{m}$ for every $q \in[1, p)$. In particular, $\lambda \in W^{1, q}(V)$ for every $q \in[1, p)$.

(2) The regularity of the roots is uniform. Let $\left\{P_{a_{\nu}} ; \nu \in \mathcal{N}\right\}$,

$$
P_{a_{\nu}(x)}(Z)=Z^{n}+\sum_{j=1}^{n} a_{\nu, j}(x) Z^{n-j} \in C^{k}(U)[Z], \nu \in \mathcal{N},
$$

be a family of polynomials, indexed by $\nu$ in some set $\mathcal{N}$, so that the set of coefficients $\left\{a_{\nu, j} ; \nu \in \mathcal{N}, j=1, \ldots, n\right\}$ is bounded in $C^{k}(U)$. Let $V \Subset U$. Then the set

$$
\left\{\lambda_{\nu} \in C^{0}(V) ; P_{a_{\nu}}\left(\lambda_{\nu}\right)=0 \text { on } V, \nu \in \mathcal{N}\right\}
$$

is bounded in $W^{1, q}(V)$ for every $q \in[1, p)$.

Remark 4.2. The roots of a polynomial depending on at least two parameters do in general not admit a continuous parameterization due to monodromy. For instance, the radical $\mathbb{C} \ni(x+i y) \mapsto(x+i y)^{1 / n}$ does not admit continuous parameterizations on $\mathbb{C}$.

Proof of Theorem 4.1. By Theorem 3.5, $\lambda$ is absolutely continuous along each affine line parallel to the coordinate axes. So $\lambda$ possesses the partial derivatives $\partial_{i} \lambda, i=1, \ldots, m$, which are defined almost everywhere and are measurable. It clearly suffices to show that all partial derivatives $\partial_{j} \lambda$ belong to $L^{q}(V)$, for every $q \in[1, p)$.

Set $x=(t, y)$, where $t=x_{1}, y=\left(x_{2}, \ldots, x_{m}\right)$, and let $V_{1}$ be the orthogonal projection of $V$ on the hyperplane $\left\{x_{1}=0\right\}$. For each $y \in V_{1}$ we denote by $V^{y}:=\{t \in \mathbb{R} ;(t, y) \in V\}$ the corresponding section of $V$; note that $V^{y}$ is open in $\mathbb{R}$. Then by Fubini's Theorem,

$$
\int_{V}\left|\partial_{1} \lambda(x)\right|^{q} d x=\int_{V_{1}} \int_{V^{y}}\left|\partial_{1} \lambda(t, y)\right|^{q} d t d y
$$

We may cover $V$ by finitely many open boxes $K=I_{1} \times \cdots \times I_{m}$ contained in $U$. Let $K$ be fixed and set $L=I_{2} \times \cdots \times I_{m}$. Fix $y \in V_{1} \cap L$ and let $\lambda_{j}^{y}, j=1, \ldots, n$, be a continuous parameterization of the roots of $P_{a}(, y)$ on $\Omega^{y}:=V^{y} \cap I_{1}$ such that $\lambda(, y)=\lambda_{1}^{y}$; it exists since $\lambda(, y)$ can be completed to a continuous parameterization of the roots of $P_{a}(, y)$ on each connected component of $\Omega^{y}$ by Lemma 6.17 of [24]. Our goal is to bound

$$
\left\|\partial_{t} \lambda(, y)\right\|_{L^{q}\left(\Omega^{y}\right)}=\left\|\left(\lambda_{1}^{y}\right)^{\prime}\right\|_{L^{q}\left(\Omega^{y}\right)}
$$

uniformly with respect to $y \in V_{1} \cap L$. 
To this end let $\mathcal{C}^{y}$ denote the set of connected components $J$ of the open set $\Omega^{y}$. For each $J \in \mathcal{C}^{y}$ extend the parameterization $\left.\lambda_{j}^{y}\right|_{J}, j=1, \ldots, n$, continuously to $I_{1}$, i.e., choose

$$
\begin{gathered}
\lambda_{j}^{y, J} \in C^{0}\left(I_{1}\right), \quad j=1, \ldots, n, \quad \text { so that } \\
\left.\forall j \quad \lambda_{j}^{y, J}\right|_{J}=\left.\lambda_{j}^{y}\right|_{J} \quad \text { and } \quad P_{a}(t, y)(Z)=\prod_{j=1}^{n}\left(Z-\lambda_{j}^{y, J}(t)\right), t \in I_{1} .
\end{gathered}
$$

This is possible since $\left.\lambda_{j}^{y}\right|_{J}$ has a continuous extension to the endpoints of the (bounded) interval $J$, by Lemma 4.3 of [14], and can then be extended on the left and on the right of $J$ by a continuous parameterization of the roots of $P_{a}(, y)$ on $I_{1}$ after suitable permutations.

By Theorem 3.5, for each $y \in V_{1} \cap L$, each $J \in \mathcal{C}^{y}$, and each $j=1, \ldots, n, \lambda_{j}^{y, J}$ is absolutely continuous on $I_{1}$ and $\left(\lambda_{j}^{y, J}\right)^{\prime} \in L^{q}\left(I_{1}\right)$ with

$$
\sup _{y, J, j}\left\|\left(\lambda_{j}^{y, J}\right)^{\prime}\right\|_{L^{q}\left(I_{1}\right)}<\infty
$$

Let $J, J_{0} \in \mathcal{C}^{y}$ be arbitrary. By Lemma 3.6. $\left(\lambda_{j}^{y}\right)^{\prime}$ as well as $\left(\lambda_{j}^{y, J_{0}}\right)^{\prime}$ belong to $L^{q}(J)$ and we have

$$
\sum_{j=1}^{n}\left\|\left(\lambda_{j}^{y}\right)^{\prime}\right\|_{L^{q}(J)}^{q}=\sum_{j=1}^{n}\left\|\left(\lambda_{j}^{y, J}\right)^{\prime}\right\|_{L^{q}(J)}^{q}=\sum_{j=1}^{n}\left\|\left(\lambda_{j}^{y, J_{0}}\right)^{\prime}\right\|_{L^{q}(J)}^{q} .
$$

Thus, for arbitrary fixed $J_{0} \in \mathcal{C}^{y}$,

$$
\begin{aligned}
\sum_{j=1}^{n}\left\|\left(\lambda_{j}^{y}\right)^{\prime}\right\|_{L^{q}\left(\Omega^{y}\right)}^{q} & =\sum_{J \in \mathcal{C}^{y}} \sum_{j=1}^{n}\left\|\left(\lambda_{j}^{y}\right)^{\prime}\right\|_{L^{q}(J)}^{q} \\
& =\sum_{J \in \mathcal{C}^{y}} \sum_{j=1}^{n}\left\|\left(\lambda_{j}^{y, J_{0}}\right)^{\prime}\right\|_{L^{q}(J)}^{q} \\
& =\sum_{j=1}^{n}\left\|\left(\lambda_{j}^{y, J_{0}}\right)^{\prime}\right\|_{L^{q}\left(\Omega^{y}\right)}^{q} \\
& \leq \sum_{j=1}^{n}\left\|\left(\lambda_{j}^{y, J_{0}}\right)^{\prime}\right\|_{L^{q}\left(I_{1}\right)}^{q}
\end{aligned}
$$

In view of (4.2) we may conclude that $\sup _{y \in V_{1} \cap L}\left\|\left(\lambda_{1}^{y}\right)^{\prime}\right\|_{L^{q}\left(\Omega^{y}\right)}<\infty$. By (4.1) and since the number of boxes $K$ is finite, this implies that $\partial_{1} \lambda \in L^{q}(V)$. The other partial derivatives can be treated analogously. This shows (1).

In order to see (2) it suffices to repeat the proof of (1) paying attention to the additional dependence on $\nu$. 


\section{Part 2. Formulas for the roots. Proof of Theorem 1.6}

\section{Strategy of the Proof}

The main ideas of the proof of Theorem 1.6 are the following. Let $x=\left(x_{1}, \ldots, x_{r}\right)$ be local coordinates at $0 \in \mathbb{C}^{r}$. Suppose that $a_{i} \in \mathbb{C}\{x\}$ and let

$$
P_{a}(Z)=Z^{n}+\sum_{j=1}^{n} a_{j} Z^{n-j}
$$

Thus we may consider $\mathscr{D}_{m}$, defined in Section 1, as an ideal of $\mathbb{C}\{x\}$. If $a_{1}=0$ and $\mathscr{D}_{2}$ is principal and generated by a monomial, then we may split $P_{a}$, that is factor it $P_{a}=P_{b} P_{c}$; see Step 2 of the proof, Section 9. This requires introducing fractional powers. If we can continue this process by splitting $P_{b}, P_{c}$, and then their factors, etc., then we eventually arrive at linear factors (i.e. of degree 1) whose coefficients are the roots. As we show in the next three sections this can be guaranteed by the principalization of the higher order discriminant ideals $\mathscr{D}_{m}$.

A subtle point of Theorem [1.6 and hence of its proof is to obtain the exponent $\alpha_{m}$ in (1.6) strictly positive. This forces us to blow-up the ideals $\mathscr{D}_{m}$ one by one. Then we put each factor in Tschirnhausen form, which amounts to subtracting a fraction of its first coefficient from the roots. The remaining part of the roots vanishes on $V\left(\mathscr{D}_{m+1}\right)$ and hence we may continue.

This consecutive splitting process can be compared to the proof of the Abhyankar-Jung Theorem of [19], that gives a formula for the roots in one shot by making the discriminant normal crossing, but without the property $\alpha_{m}>0$ which is crucial for us. In the splitting process of [19], at each stage, the coefficients of the factors, say defined on $M_{m+1}$, are expressed in terms of their product, which is well-defined on $M_{m}$. The complexity of our proof comes from the fact that, in the formula (1.6), we need each $f_{m}$ to be the pull-back of a polynomial in the coefficients $a_{i}$, that is, of a polynomial defined on $\mathbb{C}^{n}$. Similarly, each $y_{m, i}$ has to be the pull-back of a rational function on $\mathbb{C}^{n}$.

\section{A CharaCterization OF PRINCIPAlity}

Let $x=\left(x_{1}, \ldots, x_{r}\right)$ be local coordinates at $0 \in \mathbb{C}^{r}$. Suppose that $a_{i} \in \mathbb{C}\{x\}$, for $i=$ $1, \ldots, n$. We denote by $\xi(x)=\left\{\xi_{1}(x), \ldots, \xi_{n}(x)\right\}$ the unordered set of roots of $P_{a}$. Let

$$
\mathrm{S}_{m}(x)=\max _{|I|=m} \prod_{i \neq j \in I}\left|\xi_{i}(x)-\xi_{j}(x)\right| .
$$

By definition $\mathrm{S}_{m}$ is the germ at the origin of a non-negative real-valued function of $x \in \mathbb{C}^{r}$.

\section{Proposition 6.1.}

(1) There is a finite family $g_{1}, \ldots, g_{p} \in \mathscr{D}_{m}$ such that $\mathrm{S}_{m}^{N ! / m(m-1)} \sim \max _{j}\left|g_{j}\right|$. Moreover, we may take as $g_{1}, \ldots, g_{p}$ any system of generators of $\mathscr{D}_{m}$.

(2) $g \in \mathscr{D}_{m}$ generates $\mathscr{D}_{m}$ if and only if $\mathrm{S}_{m}^{N ! / m(m-1)} \sim|g|$. 
Proof. (1) We can choose as $g_{1}, \ldots, g_{p}$ the powers $\sigma_{i}^{N ! / i m(m-1)}$ of the elementary symmetric functions $\sigma_{i}, i=1, \ldots,\left(\begin{array}{c}n \\ m\end{array}\right)$, in $\lambda_{I}=\prod_{i \neq j \in I}\left(\xi_{i}(x)-\xi_{j}(x)\right), I \subset\{1, \ldots, n\},|I|=m$. Indeed

$$
\left.\left.\left|\sigma_{s}\left(\lambda_{I} ;|I|=m\right)\right|^{\frac{N !}{s m(m-1)}} \leq\left(\begin{array}{c}
n \\
m
\end{array}\right)\right)^{\frac{N !}{s m(m-1)}} \max _{I}\left|\lambda_{I}\right|^{\frac{N !}{m(m-1)}}=\left(\begin{array}{c}
n \\
m
\end{array}\right)\right)^{\frac{N !}{s m(m-1)}} \mathrm{S}_{m}(x)^{\frac{N !}{m(m-1)}}
$$

The converse estimate follows from Lemma 6.2 below.

(2) By (1) if $g$ generates $\mathscr{D}_{m}$ then $|g| \sim \mathrm{S}_{m}^{N ! / m(m-1)}$. Suppose now that $|g| \sim \mathrm{S}_{m}^{N ! / m(m-1)}$. Then for any $f \in \mathscr{D}_{m}$ the quotient $f / g$ is bounded and hence holomorphic.

Lemma 6.2 ([16] p. 56, or [21] Theorem 1.1.4.). If $a_{1}, \ldots, a_{n}, z \in \mathbb{C}$ satisfy the equation $z^{n}+\sum_{j=1}^{n} a_{j} z^{n-j}=0$, then $|z| \leq 2 \max _{j}\left|a_{j}\right|^{1 / j}$.

Corollary 6.3. If $\mathscr{D}_{m}$ is principal then $\mathscr{D}_{l} \subset \mathscr{D}_{m}$ for $l \geq m$.

Corollary 6.4. The zero set of $\mathscr{D}_{m}$ equals $\left\{x ;\left|\left\{\xi_{1}(x), \ldots, \xi_{n}(x)\right\}\right|<m\right\}$.

Corollary 6.5. Suppose that $a_{1}=0$. Then $\mathscr{D}_{2}$ is principal if and only if so is $\left(a_{2}^{N ! / 2}, \ldots, a_{n}^{N ! / n}\right)$, and then both ideals coincide.

Thus, if $\mathscr{D}_{2}=(g)$ then $g$ divides each $a_{i}^{N ! / i}$ and there is $i_{0}$ such that $|g| \sim\left|a_{i_{0}}^{N ! / i_{0}}\right|$.

Proof. Shortly speaking, this corollary follows from the fact that, by Proposition 1.2, the ideals $\mathscr{D}_{2}$ and $\left(a_{2}^{N ! / 2}, \ldots, a_{n}^{N ! / n}\right)$ have the same integral closure.

More precisely, in the Tschirnhausen case $a_{1}=0$,

$$
\begin{gathered}
\max _{j}\left|\xi_{j}\right|=\max _{j}\left|\frac{1}{n} \sum_{k}\left(\xi_{j}-\xi_{k}\right)\right| \leq \max _{j} \frac{1}{n} \sum_{k}\left|\xi_{j}-\xi_{k}\right| \leq \max _{k \neq j}\left|\xi_{j}-\xi_{k}\right|, \\
\max _{k \neq j}\left|\xi_{j}-\xi_{k}\right| \leq \max _{k \neq j}\left(\left|\xi_{j}\right|+\left|\xi_{k}\right|\right) \leq 2 \max _{j}\left|\xi_{j}\right|,
\end{gathered}
$$

and hence, by Lemma 6.2 ,

$$
\mathrm{S}_{2} \sim \max _{i}\left|\xi_{i}\right|^{2} \sim \max _{j}\left|a_{j}\right|^{2 / j}
$$

Thus if $a_{i}^{N ! / i}$ generates $\left(a_{2}^{N ! / 2}, \ldots, a_{n}^{N ! / n}\right)$ then for any $f \in \mathscr{D}_{2}, f / a_{i}^{N ! / i}$ is bounded and hence holomorphic. Therefore $a_{i}^{N ! / i}$ generates $\mathscr{D}_{2}$.

Conversely, if $g$ generates $\mathscr{D}_{2}$ then by (6.1) one of the $a_{i}^{N ! / i} / g$ does not vanish at the origin and hence $g \in\left(a_{2}^{N ! / 2}, \ldots, a_{n}^{N ! / n}\right)$.

\section{Convexity}

For a power series in one variable $t, \lambda \in \mathbb{C}\{t\}$, we define its order $\operatorname{ord}_{0} \lambda$ as the leading exponent $\lambda(t)=a_{0} t^{\operatorname{ord}_{0} \lambda}+\cdots$ and set $\operatorname{ord}_{0} 0:=\infty$. Given power series $\lambda_{i} \in \mathbb{C}\{t\}, i=$ $1, \ldots, n$, we define for $2 \leq m \leq n$

$$
\alpha(m):=\min _{|I|=m} \operatorname{ord}_{0} \prod_{i \neq j \in I}\left(\lambda_{i}-\lambda_{j}\right) .
$$

Proposition 7.1. For $3 \leq m \leq n-1$

$$
2 \alpha(m)+\alpha(2) \leq \alpha(m-1)+\alpha(m+1) .
$$


Example 7.2. If all $\lambda_{i}-\lambda_{j}$ have the same order, say equal to 1 , then $\alpha(m)=2\left(\begin{array}{c}m \\ 2\end{array}\right)$ and we have equality.

For the proof we first make some reduction. By shifting all $\lambda_{i}$ by $\frac{1}{n} \sum_{i} \lambda_{i}$ we may assume that $\sum_{i} \lambda_{i}=0$. Then $\alpha(2)=2 \min _{i} \operatorname{ord}_{0} \lambda_{i}$. Indeed,

$$
\begin{aligned}
\alpha(2) / 2 & =\min _{i \neq j} \operatorname{ord}_{0}\left(\lambda_{i}-\lambda_{j}\right) \geq \min _{i \neq j} \min \left\{\operatorname{ord}_{0} \lambda_{i}, \operatorname{ord}_{0} \lambda_{j}\right\}=\min _{i} \operatorname{ord}_{0} \lambda_{i} \\
& =\min _{i} \operatorname{ord}_{0} \frac{1}{n} \sum_{k}\left(\lambda_{i}-\lambda_{k}\right) \geq \min _{i} \min _{i \neq k} \operatorname{ord}_{0}\left(\lambda_{i}-\lambda_{k}\right)=\alpha(2) / 2 .
\end{aligned}
$$

Thus, by dividing all $\lambda_{i}$ by $t^{\alpha(2) / 2}$, we may suppose that $\alpha(2)=0$. Then (7.1) becomes a genuine convexity relation.

Let us divide the set of $\lambda_{i}$ into the union of two disjoint non-empty subsets $\left\{\lambda_{i} ; i \in I\right\}$ and $\left\{\lambda_{j} ; i \in J\right\},|I|=n_{1}<n,|J|=n_{2}<n, n_{1}+n_{2}=n$, so that for each $i \in I, j \in J$, $\operatorname{ord}_{0}\left(\lambda_{i}-\lambda_{j}\right)=0$. We shall call such a partition a splitting.

The corresponding orders for these two families we denote by $\beta(m), 2 \leq m \leq n_{1}$, and $\gamma(m), 2 \leq m \leq n_{2}$. Then for each $2 \leq m \leq n$ there is $0 \leq m_{1} \leq n_{1}$ such that

$$
\alpha(m)=\beta\left(m_{1}\right)+\gamma\left(m-m_{1}\right) .
$$

It is possible that $m_{1}$ or $m-m_{1}$ is equal to 0 or 1 . In this case we put $\beta(0)=\beta(1)=\gamma(0)=$ $\gamma(1)=0$.

For a couple of integers $a \leq b$ we denote by $[a \ldots b]:=\{c \in \mathbb{Z} ; a \leq c \leq b\}$ the set of all integers between $a$ and $b$ and call it an interval.

Proposition 7.3. For each $m$ the set of $m_{1}$ such that (7.2) holds is an interval. If we denote this interval by $\left[\underline{K}_{1}(m) . . \bar{K}_{1}(m)\right]$ then, for $2 \leq m \leq n-1, \underline{K}_{1}(m) \leq \underline{K}_{1}(m+1) \leq \underline{K}_{1}(m)+1$ and $\bar{K}_{1}(m) \leq \bar{K}_{1}(m+1) \leq \bar{K}_{1}(m)+1$.

Proof of Propositions 7.1 and 7.3. First we show Proposition 7.3 assuming Proposition 7.1 for $\beta$ and $\gamma$. Let

$$
\varphi_{m}\left(m_{1}\right):=\beta\left(m_{1}\right)+\gamma\left(m-m_{1}\right) .
$$

The set of $m_{1}$ such that (7.2) holds is the set of $m_{1}$ at which $\varphi_{m}\left(m_{1}\right)$ is minimal. It is an interval since, by assumption, $\varphi_{m}\left(m_{1}\right)$ is convex. Moreover, by convexity, $\varphi_{m}$ is decreasing on $\left[2 \ldots \underline{K}_{1}(m)\right]$ and increasing on $\left[\bar{K}_{1}(m) . . n_{1}\right]$.

We show that it is not possible that $\varphi_{m}\left(m_{1}\right) \leq \varphi_{m}\left(m_{1}+1\right)$ and $\varphi_{m+1}\left(m_{1}+1\right) \geq \varphi_{m+1}\left(m_{1}+\right.$ 2 ) with one of these inequalities strict. Indeed, if this were the case then

$$
\begin{gathered}
\varphi_{m}\left(m_{1}\right)+\varphi_{m+1}\left(m_{1}+2\right)=\beta\left(m_{1}\right)+\gamma\left(m-m_{1}\right)+\beta\left(m_{1}+2\right)+\gamma\left(m-m_{1}-1\right) \\
<\varphi_{m}\left(m_{1}+1\right)+\varphi_{m+1}\left(m_{1}+1\right)=2 \beta\left(m_{1}+1\right)+\gamma\left(m-m_{1}-1\right)+\gamma\left(m-m_{1}\right)
\end{gathered}
$$

which contradicts the convexity of $\beta$. This implies that $\bar{K}_{1}(m+1) \leq \bar{K}_{1}(m)+1$ and $\underline{K}_{1}(m+1) \leq \underline{K}_{1}(m)+1$. By interchanging $I, \beta$ and $J, \gamma$ we obtain $\bar{K}_{2}(m+1) \leq \bar{K}_{2}(m)+1$ and $\underline{K}_{2}(m+1) \leq \underline{K}_{2}(m)+1$ which are the desired inequalities in view of $\bar{K}_{2}(m)=m-\underline{K}_{1}(m)$ and $\underline{K}_{2}(m)=m-\bar{K}_{1}(m)$, where $\underline{K}_{2}, \bar{K}_{2}$ play the roles of $\underline{K}_{1}, \bar{K}_{1}$ for $\gamma$.

Now we show how Proposition 7.3 implies Proposition 7.1 for $\alpha$. Fix $m$ such that $3 \leq m \leq$ $n-1$. By Proposition 7.3 we may assume $\left[\underline{K}_{1}(m-1) . . \bar{K}_{1}(m-1)\right] \cap\left[\underline{K}_{1}(m) . . \bar{K}_{1}(m)\right] \neq \emptyset$. 
(If this is not the case then $\underline{K}_{1}(m)=\bar{K}_{1}(m)=\underline{K}_{1}(m-1)+1=\bar{K}_{1}(m-1)+1$ and thus $\underline{K}_{2}(m-1)=\bar{K}_{2}(m-1)=\underline{K}_{2}(m)=\bar{K}_{2}(m)$.)

Fix $m_{1} \in\left[\underline{K}_{1}(m-1) . . \bar{K}_{1}(m-1)\right] \cap\left[\underline{K}_{1}(m) . . \bar{K}_{1}(m)\right]$. Then, by Proposition 7.3, either $m_{1}$ or $m_{1}+1$ belongs to $\left[\underline{K}_{1}(m+1) . . \overline{K_{1}}(m+1)\right]$. In the former case

$$
\begin{aligned}
\alpha(m-1)+\alpha(m+1) & =\beta\left(m_{1}\right)+\gamma\left(m-m_{1}-1\right)+\beta\left(m_{1}\right)+\gamma\left(m-m_{1}+1\right) \\
& \geq 2\left(\beta\left(m_{1}\right)+\gamma\left(m-m_{1}\right)\right)=2 \alpha(m) .
\end{aligned}
$$

In the latter case

$$
\begin{aligned}
\alpha(m-1)+\alpha(m+1) & =\beta\left(m_{1}\right)+\gamma\left(m-m_{1}-1\right)+\beta\left(m_{1}+1\right)+\gamma\left(m-m_{1}\right) \\
& \geq 2\left(\beta\left(m_{1}\right)+\gamma\left(m-m_{1}\right)\right)=2 \alpha(m),
\end{aligned}
$$

since $\beta\left(m_{1}+1\right)+\gamma\left(m-m_{1}-1\right) \geq \beta\left(m_{1}\right)+\gamma\left(m-m_{1}\right)$ as $m_{1} \in\left[\underline{K}_{1}(m) . . \bar{K}_{1}(m)\right]$.

\section{Splitting}

We suppose that $a_{i} \in \mathbb{C}\{x\}, x=\left(x_{1}, \ldots, x_{r}\right)$, and that $P_{a}$ factors

$$
P_{a}(Z)=P_{b}(Z) P_{c}(Z)
$$

where $P_{b}(Z)=Z^{n_{1}}+b_{1} Z^{n_{1}-1}+\cdots+b_{n_{1}}, P_{c}(Z)=Z^{n_{2}}+c_{1} Z^{n_{2}-1}+\cdots+c_{n_{2}}, b_{i}, c_{j} \in \mathbb{C}\{x\}$, $n=n_{1}+n_{2}, n_{1}, n_{2}>0$.

Assumption. We shall assume that the resultant of $P_{b}$ and $P_{c}$ does not vanish at 0 , that is $P_{b(0)}$ and $P_{c(0)}$ do not have common roots.

In order to distinguish the ideals $\mathscr{D}_{m}$ for polynomials $P_{a}, P_{b}$, and $P_{c}$ we shall denote them by $\mathscr{D}_{a, m}, \mathscr{D}_{b, m}$, and $\mathscr{D}_{c, m}$ respectively; likewise for the size functions $\mathrm{S}_{a, m}, \mathrm{~S}_{b, m}, \mathrm{~S}_{c, m}$.

\section{Proposition 8.1.}

(1) Suppose that $\mathscr{D}_{a, m}$ is a principal ideal. Then there are $m_{1} \geq 0, m_{2} \geq 0, m_{1}+m_{2}=m$, such that

$$
\mathrm{S}_{a, m} \sim \mathrm{S}_{b, m_{1}} \mathrm{~S}_{c, m_{2}},
$$

and for any such $m_{1}, m_{2}, \mathscr{D}_{b, m_{1}}$ and $\mathscr{D}_{c, m_{2}}$ are principal. $\left(\right.$ We put $S_{0}=S_{1}=1$.) Moreover, the set of those $m_{1}$ for which (8.1) holds, with $m_{2}=m-m_{1}$, is an interval. We denote this interval by $\left[\underline{K}_{1}(m) . . \bar{K}_{1}(m)\right]$.

(2) If the ideals $\mathscr{D}_{a, m}$ and $\mathscr{D}_{a, m+1}$ are principal then $\underline{K}_{1}(m) \leq \underline{K}_{1}(m+1) \leq \underline{K}_{1}(m)+1$ and $\bar{K}_{1}(m) \leq \bar{K}_{1}(m+1) \leq \bar{K}_{1}(m)+1$.

(3) Suppose that the ideals $\mathscr{D}_{a, i}$ are principal for $2 \leq i \leq m$ and that (8.1) holds. Then $\mathscr{D}_{b, i}$ and $\mathscr{D}_{c, j}$ are principal for all $i \leq m_{1}$ and $j \leq m_{2}$.

Proof. For fixed $x$ we may order the roots of $P_{a(x)}$. Given $I \subset\{1, \ldots, n\},|I|=m$, we divide $I=I^{\prime} \cup I^{\prime \prime}$, so that $I^{\prime},\left|I^{\prime}\right|=m_{1}$, labels the roots of $P_{b(x)}$ and $I^{\prime \prime},\left|I^{\prime \prime}\right|=m_{2}$, the roots of $P_{c(x)}$. Then

$$
\prod_{i, j \in I, i \neq j}\left(\xi_{i}(x)-\xi_{j}(x)\right)=\prod_{i \neq j \in I^{\prime}}\left(\xi_{i}(x)-\xi_{j}(x)\right) \cdot \prod_{i \neq j \in I^{\prime \prime}}\left(\xi_{i}(x)-\xi_{j}(x)\right) \cdot \varphi(x),
$$


and $\varphi(x)=\prod_{i \in I^{\prime}, j \in I^{\prime \prime}}\left(\xi_{i}(x)-\xi_{j}(x)\right)$ is non-zero and close to $\prod_{i \in I^{\prime}, j \in I^{\prime \prime}}\left(\xi_{i}(0)-\xi_{j}(0)\right)$. Therefore, as functions of $x$,

$$
\mathrm{S}_{a, m} \sim \max _{m_{1}+m_{2}=m} \mathrm{~S}_{b, m_{1}} \mathrm{~S}_{c, m_{2}}
$$

Suppose that $\mathscr{D}_{a, m}$ is generated by $f$. By (8.2) there exist $m_{1}+m_{2}=m$ and $h \in \mathscr{D}_{b, m_{1}}$, $g \in \mathscr{D}_{c, m_{2}}$ such that $(g h / f)(0) \neq 0$. Then (8.1) holds and, by Proposition 6.1, $g$ generates $\mathscr{D}_{b, m_{1}}$ and $h$ generates $\mathscr{D}_{c, m_{2}}$.

Thus if $\mathscr{D}_{a, m}$ is principal then the set of $m_{1}$ for which (8.1) holds, which we denote by $\mathcal{M}$, is non-empty. By the curve selection lemma, see for instance [18], (8.1) holds if and only if it holds on every real analytic curve $x(t):(\mathbb{R}, 0) \rightarrow\left(\mathbb{C}^{r}, 0\right)$. Therefore, by (8.1) and Proposition 7.3. $\mathcal{M}$, as an intersection of intervals, is an interval. Denote it by $\left[\underline{K}_{1}(m) . . \bar{K}_{1}(m)\right]$. Moreover, if $\mathscr{D}_{a, m}$ and $\mathscr{D}_{a, m+1}$ are principal then $\underline{K}_{1}(m), \bar{K}_{1}(m)$ satisfy the inequalities of Proposition 7.3 . Indeed, suppose for instance that $\bar{K}_{1}(m) \leq \bar{K}_{1}(m+1)$ fails. Then there exists a real analytic curve on which $\mathrm{S}_{a, m+1} \sim \mathrm{S}_{b, m_{1}} \mathrm{~S}_{c, m+1-m_{1}}$ with $m_{1}=\bar{K}_{1}(m)$ fails but is satisfied for some $m_{1}<\bar{K}_{1}(m)$. But this contradicts Proposition 7.3 since (8.1) holds on this curve. This shows (1) and (2).

Finally (3) follows from (1) and (2).

Proposition 8.2. Suppose that $\mathscr{D}_{a, i}$ are principal for all $2 \leq i \leq n$. Let $m_{1}(m), m_{2}(m)=$ $m-m_{1}(m)$ be two non-decreasing integer-valued functions defined for $0 \leq m \leq n$, such that $0 \leq m_{1}(m) \leq n_{1}, 0 \leq m_{2}(m) \leq n_{2}$, for every $0 \leq m \leq n$, and such that (8.1) holds for each triple $\left(m, m_{1}(m), m_{2}(m)\right), 0 \leq m \leq n$. Let $\mathscr{D}_{a, m}=\left(f_{m}\right), \mathscr{D}_{b, m_{1}}=\left(g_{m_{1}}\right), \mathscr{D}_{c, m_{2}}=\left(h_{m_{2}}\right)$.

Then, for every $1 \leq m \leq n$ exactly one of the following two cases happens

(1) $m_{1}(m)=m_{1}(m-1)+1, m_{2}(m)=m_{2}(m-1)$.

(2) $m_{2}(m)=m_{2}(m-1)+1, m_{1}(m)=m_{1}(m-1)$.

Moreover, if (1) holds then $g_{m_{1}}\left|f_{m}\right| g_{m_{1}}^{m}$, and symmetrically, if (2) holds then $h_{m_{2}}\left|f_{m}\right| h_{m_{2}}^{m}$.

Proof. Two non-decreasing non-negative functions $m_{1}, m_{2}$ such that $m_{1}(m)+m_{2}(m)=m$ must satisfy either (1) or (2). Thus it suffices to show that if (1) is satisfied then $g_{m_{1}}\left|f_{m}\right| g_{m_{1}}^{m}$. The first condition $g_{m_{1}} \mid f_{m}$ follows easily from the proof of Proposition 8.1. If (8.1) holds then $g_{m_{1}} h_{m_{2}}$ generates $\mathscr{D}_{a, m}$ and we may suppose that

$$
f_{m}=g_{m_{1}} h_{m_{2}} \text {. }
$$

By Corollary 6.3, $1=f_{1}\left|f_{2}\right| \cdots \mid f_{n}$, and similarly $1=g_{1}\left|g_{2}\right| \cdots \mid g_{n_{1}}$ and $1=h_{1}\left|h_{2}\right| \cdots \mid h_{n_{2}}$.

Let $r$ be given by $m_{1}(m)=m_{1}(m-1)+1=m_{1}(m-2)+1=\cdots=m_{1}(m-r)+1=$ $m_{1}(m-r-1)+2$; we have Case (1) for $m$ and $m-r$ and Case (2) in between. We write $m_{1}=m_{1}(m), m_{2}=m_{2}(m)$ for short. Then

$$
\begin{aligned}
& f_{m-1}=g_{m_{1}-1} h_{m_{2}} \mid g_{m_{1}} h_{m_{2}-1} \\
& \ldots \\
& f_{m-r+1}=g_{m_{1}-1} h_{m_{2}-r+2} \mid g_{m_{1}} h_{m_{2}-r+1} \\
& f_{m-r}=g_{m_{1}-1} h_{m_{2}-r+1}
\end{aligned}
$$

Hence

$$
h_{m_{2}}\left|\left(g_{m_{1}} / g_{m_{1}-1}\right) h_{m_{2}-1}\right| \cdots \mid\left(g_{m_{1}} / g_{m_{1}-1}\right)^{r-1} h_{m_{2}-r+1} \text {. }
$$


Consequently

$$
f_{m}=g_{m_{1}} h_{m_{2}} \mid\left(g_{m_{1}} / g_{m_{1}-1}\right)^{r} g_{m_{1}-1} h_{m_{2}-r+1}=\left(g_{m_{1}} / g_{m_{1}-1}\right)^{r} f_{m-r} .
$$

Since $m_{1}(m-r)+1=m_{1}(m-r-1)+2$, by induction on $m_{1}, f_{m-r} \mid g_{m_{1}-1}^{m-r}$ (we may start the induction by formally putting $f_{1}=g_{1}=h_{1}=1$ ), which shows $f_{m} \mid g_{m_{1}}^{m}$ as $g_{m_{1}-1} \mid g_{m_{1}}$.

Remark 8.3. By Proposition 8.1, both $m_{1}(m)=\underline{K}_{1}(m)$ and $m_{1}(m)=\bar{K}_{1}(m)$ satisfy the assumptions of Proposition 8.2 if we complete them by putting $\underline{K}_{1}(1)=0, \bar{K}_{1}(1)=1$. In particular, there exists a function $m_{1}(m)$ satisfying the assumptions of Proposition 8.2, such that $m_{1}(2)=m_{2}(2)=1$. This follows from the assumption that $P_{b(0)}$ and $P_{c(0)}$ do not have common roots; in particular, not all roots of $P_{a(0)}$ coincide and thus $\mathrm{S}_{a, 2} \sim 1$.

Fix such a function $m_{1}(m)$. For $2 \leq m_{1} \leq n_{1}$ define $m_{b}\left(m_{1}\right)$ as the smallest $m$ such that $m_{1}=m_{1}(m)$, i.e. $m_{b}\left(m_{1}\right)$ as a function is the lowest inverse of $m_{1}(m)$. Similarly we define $m_{c}\left(m_{2}\right)$ for $2 \leq m_{2} \leq n_{2}$. The functions $m_{b}$ and $m_{c}$ are strictly increasing and each $3 \leq m \leq n$ is in the image of precisely one of them. See the example in Figure 1.

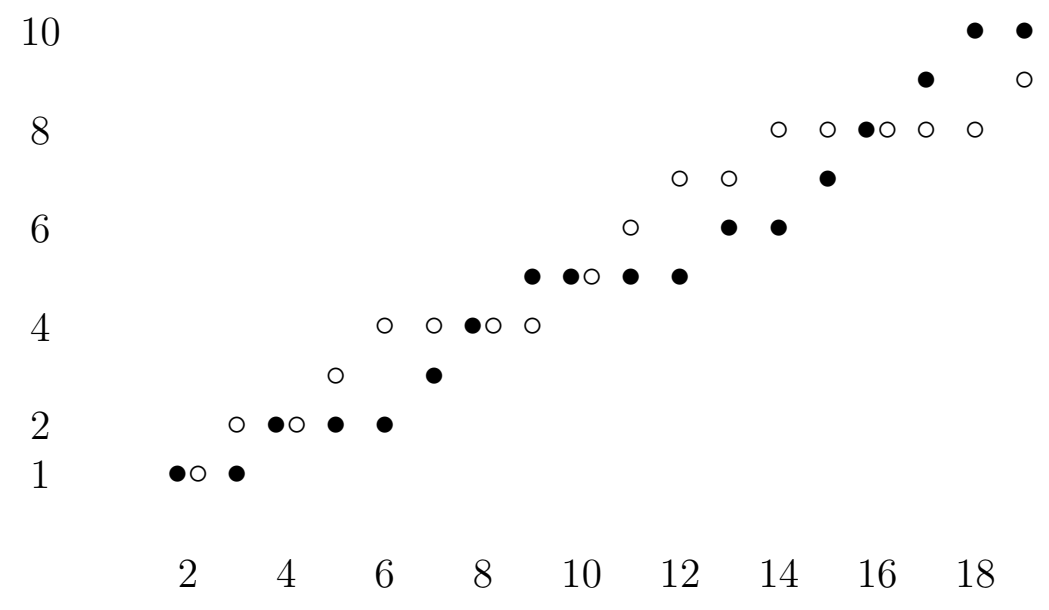

Figure 1. An example for the functions $m_{1}(m)$, bullets $\bullet$, and $m_{2}(m)$, circles $\circ$. In this example $\left(m_{b}(2), m_{b}(3), \ldots\right)=(4,7,8,9,13,15,16,17,18)$ and $\left(m_{c}(2), m_{c}(3), \ldots\right)=(3,5,6,10,11,12,14,19)$; these are the points where the respective sequence increases by 1 . The two sets form a partition of the integers between 3 and 19 .

\section{Proof of Theorem 1.6}

Proof. We first put $P_{a}$ into Tschirnhausen form (1.2), then split it into two factors after blowing up $\mathscr{D}_{2}$. These factors are defined locally on the blow-up space $M_{2}$. Then we put each of these factors into Tschirnhausen form and continue the process by splitting the subsequent factors on $M_{3}, M_{4}$ and so on, putting first every new factor into Tschirnhausen form. At each stage the Tschirnhausen transformation shifts the roots exactly by the term $A_{m} \varphi_{m}$ of (1.5). 
In order to simplify the notation we use the same letters for the functions and their pullbacks to the blow-up spaces, for instance we write $a_{k}$ for $a_{k} \circ \sigma_{m}$.

Step 1. First we perform the Tschirnhausen transformation by replacing $Z$ by $Z-a_{1} / n$ and hence shifting the roots by $a_{1} / n$. Thus we put

$$
\varphi_{1}:=a_{1} \quad A_{1}:=\frac{1}{n} .
$$

(Recall that the Tschirnhausen transformation does not change the ideals $\mathscr{D}_{m}$.) After this transformation we may assume that $P_{a}(Z)$ is in the Tschirnhausen form (1.2).

Step 2. Fix $p_{2} \in M_{2}$ and a privileged system of coordinates $y_{2,1}, \ldots, y_{2, n}$ at $p_{2}$. We shall split $P_{a}$ at $p_{2}$

$$
P_{a}=P_{b} P_{c}
$$

where $P_{b}(Z)=Z^{n_{1}}+\sum_{i=1}^{n_{1}} b_{i} Z^{n_{1}-i}, P_{c}(Z)=Z^{n_{2}}+\sum_{j=1}^{n_{2}} c_{j} Z^{n_{2}-j}$, with $n_{1}>0, n_{2}>0$, $b_{i}, c_{j} \in \mathbb{C}\left\{y_{2,1}^{1 / q_{2}}, \ldots, y_{2, n}^{1 / q_{2}}\right\}, q_{2}=N$ !, as follows. Let $f_{2}$ generate $\mathscr{D}_{2}$ at $p_{2}$. By Corollary 6.5, $f_{2}$ divides each $a_{i}^{N ! / i}$ and there is $i_{0}$ such that $f_{2}$ equals $a_{i_{0}}^{N ! / i_{0}}$ times a unit. Consider an auxiliary polynomial

$$
Q_{\bar{a}}(\tilde{Z}):=f_{2}^{-n / q_{2}} P_{a}\left(f_{2}^{1 / q_{2}} \tilde{Z}\right)=\tilde{Z}^{n}+\bar{a}_{2} \tilde{Z}^{n-2}+\cdots+\bar{a}_{n},
$$

where $\bar{a}_{i}=f_{2}^{-i / q_{2}} a_{i}, \bar{a}_{i_{0}}\left(p_{2}\right) \neq 0$. Because $f_{2}$ is a normal crossing in $y_{2, i}$, cf. (1.3),

$$
f_{2}^{1 / q_{2}} \in \mathbb{C}\left\{\left(y_{2,1}^{1 / q_{2}}, \ldots, y_{2, r_{2}}^{1 / q_{2}}, y_{2, r_{2}+1}, \ldots, y_{2, n}\right)\right\} .
$$

We first split $Q_{\bar{a}}(\tilde{Z})=Q_{\bar{b}}(\tilde{Z}) Q_{\bar{c}}(\tilde{Z})$ using the following lemma, see e.g. [1] or [2].

Lemma 9.1. Let $Q_{a^{\prime}}=Q_{b^{\prime}} Q_{c^{\prime}}, a^{\prime}=\left(a_{1}^{\prime}, \ldots, a_{n}^{\prime}\right) \in \mathbb{C}^{n}, b^{\prime}=\left(b_{1}^{\prime}, \cdots, b_{n_{1}}^{\prime}\right) \in \mathbb{C}^{n_{1}}, c^{\prime}=$ $\left(c_{1}^{\prime}, \cdots, c_{n_{2}}^{\prime}\right) \in \mathbb{C}^{n_{2}}$, be monic complex polynomials. Suppose that $Q_{b^{\prime}}$ and $Q_{c^{\prime}}$ have no common root. Then there are complex analytic mappings $b(a), c(a)$, defined in a neighborhood of $a^{\prime}$ in $\mathbb{C}^{n}$, such that

and $b^{\prime}=b\left(a^{\prime}\right), c^{\prime}=c\left(a^{\prime}\right)$.

$$
Q_{a}=Q_{b(a)} Q_{c(a)}
$$

Proof. If we write $Q_{a}=Q_{b} Q_{c}$ and compute $a$ as a function of $b$ and $c$, denoted by $a(b, c)$, then the Jacobian determinant of $a(b, c)$ equals the resultant of $Q_{b}$ and $Q_{c}$ which is nonzero by assumption. Thus the lemma follows from the Inverse Function Theorem.

Since $\bar{a}_{i_{0}}\left(p_{2}\right) \neq 0$ and $Q_{\tilde{a}}(\tilde{Z})$ is in Tschirnhausen form, $Q_{\bar{a}\left(p_{2}\right)}(\tilde{Z})=\tilde{Z}^{n}+\bar{a}_{2}\left(p_{2}\right) \tilde{Z}^{d-2}+$ $\cdots+\bar{a}_{n}\left(p_{2}\right)$ has at least two distinct complex roots and thus can be written as the product of two factors with no common roots. Then Lemma 9.1 allows us to extend this splitting to a neighborhood of $p_{2}$

$$
Q_{\bar{a}}(\tilde{Z})=Q_{\bar{b}}(\tilde{Z}) Q_{\bar{c}}(\tilde{Z}) .
$$

This splitting induces a splitting (9.1) of $P_{a}$ by setting $P_{b}(Z):=f_{2}^{n_{1} / q_{2}} Q_{\bar{b}}\left(f_{2}^{-1 / q_{2}} Z\right), n_{1}=$ $\operatorname{deg} Q_{\bar{b}}$, that is

$$
b_{i}:=f_{2}^{i / q_{2}} \eta_{i}\left(y_{2,1}^{1 / q_{2}}, \ldots, y_{2, r_{2}}^{1 / q_{2}}, y_{2, r_{2}+1}, \ldots, y_{2, n}\right), \quad i=1, \ldots, n_{1}=\operatorname{deg} P_{b},
$$


where $\eta_{i}$ is a convergent power series. Similar formulas hold for $P_{c}$. The next step involves putting both $P_{b}$ and $P_{c}$ in Tschirnhausen form. Thus if we put

$$
\varphi_{2}:=b_{1}=-c_{1}
$$

then by the Tschirnhausen transformation the roots of $P_{b}$ are shifted by $-\frac{\varphi_{2}}{n_{1}}$ and those of $P_{c}$ by $\frac{\varphi_{2}}{n_{2}}$.

Step 3. Let $\mathrm{C}=\left(p_{m}, f_{m}, h_{m}, P_{m, i}, s_{m}, r_{m}\right)$ be a chain for $p_{n} \in M_{n}$ with neighborhoods $\mathcal{U}_{m}$ of $p_{m}$. From $\mathrm{C}$ we extract the chains for $P_{b}$ and $P_{c}$ as follows. Choose the functions $m_{1}(m)$, $m_{2}(m), m_{b}\left(m_{1}\right), m_{c}\left(m_{2}\right)$ as in Remark 8.3 and split the chain

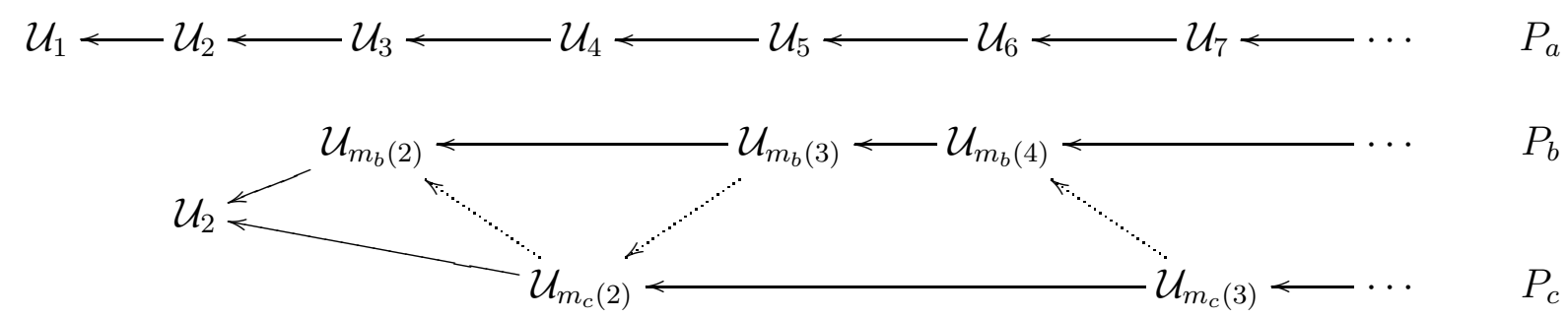

We claim that by Propositions 8.1 and 8.2

$$
\mathcal{U}_{2} \longleftarrow \mathcal{U}_{m_{b}(2)} \longleftarrow \cdots \longleftarrow \mathcal{U}_{m_{b}\left(n_{1}\right)},
$$

defines a chain for $p_{n}$ associated with $P_{b}$. Indeed, the pull-back of $\mathscr{D}_{b, m_{1}}$ on $\left(\mathcal{U}_{m}, p_{m}\right), m=$ $m_{b}\left(m_{1}\right)$, is invertible and generated by a (fractional) normal crossing in $y_{m, 1}, \ldots, y_{m, r_{m}}$, this generator is denoted $g_{m_{1}}$ in Proposition 8.2. The rest of the data defining the chain $\left(h_{m}, P_{m, i}, s_{m}, r_{m}\right)$ is the same.

Then, if $m=m_{b}\left(m_{1}\right) \geq 2$, we set $\varphi_{m}:=\varphi_{b, m_{1}}$. By Proposition 8.2

$$
\varphi_{m}=g_{m_{1}}^{\alpha_{b, m_{1}}} \psi_{b, m_{1}}=f_{m}^{\alpha_{b, m_{1}} / m} \psi_{m},
$$

(this equation defines $\psi_{m}$ ). By the inductive assumption every root of $P_{b}$ is a combination of the $\varphi_{b, i}$. Since each root of $P_{a}$ is either a root of $P_{b}$ or of $P_{c}$, the proof is complete.

9.1. Proof of Lemma 1.9. We prove Lemma 1.9 following closely the steps of the proof of Theorem 1.6.

Step 1. Clearly $\varphi_{1}(t)=a_{1}(t)$. Thus after a shift of $\lambda(t)$ by $\frac{1}{n} \varphi_{1}(t)$ we may assume that $P_{a}(t)$ is in Tschirnhausen form.

Step 2. The crucial observation is that for all $m \geq 2, f_{m}(a(t)), y_{m, 1}\left(\widehat{a}_{m}(t)\right), \ldots, y_{m, r_{m}}\left(\widehat{a}_{m}(t)\right)$ do not vanish on $J$. Hence we may choose their $q_{m}$-th radicals continuously, and even of the same regularity $\left(C^{k}\right.$, real analytic, etc.) as the coefficients $a_{i}(t)$.

Thus a root $\lambda(t)$ of $P_{a(t)}$ induces a root $f_{2}^{-\alpha_{2}}(t) \lambda(t)$ of $Q_{\bar{a}(t)}$, whose coefficients are now well-defined as functions of $t \in J$. Since the roots of $Q_{\bar{b}(t)}, Q_{\bar{c}(t)}$, are distinct, $f_{2}^{-\alpha_{2}}(t) \lambda(t)$ is a root of precisely one of them. Thus we may consider $\lambda(t)$ as a root of $P_{b(t)}$ for instance.

Step 3. Then, on $M_{m}, m=m_{b}(2)$, perform the Tschirnhausen transformation of $P_{b(t)}$, split it and by choosing the radical $g_{m_{1}}^{\alpha_{b, m_{1}}}(t)$ identify $\lambda(t)$ (shifted by the Tschirnhausen transformation) with a root of one of these factors. We continue these procedure until the last factor is of degree 1. 
We note that on $J$ we have $n$ everywhere distinct continuous roots of $P_{a(t)}$. They separate in the above process; any two of them, shifted first by common Tschirnhausen transformations, are roots of different factors at some stage.

\section{Part 3. Example. Roots of cubic polynomials.}

\section{Statement of Result.}

We give a detailed presentation of the degree 3 case as an example. In this case the resolution is explicit and the result can be made more precise.

Theorem 10.1. Let $I \subset \mathbb{R}$ be a bounded open interval. Consider a monic polynomial

$$
P(t)(Z)=Z^{3}+a_{1}(t) Z^{2}+a_{2}(t) Z+a_{3}(t), \quad t \in I,
$$

with coefficients $a_{j} \in C^{6}(\bar{I}), j=1,2,3$. Then:

(1) If $\lambda_{j}: I \rightarrow \mathbb{C}, j=1,2,3$, denotes a continuous parameterization of the roots of $P$, then each $\lambda_{j}^{\prime} \in L_{w}^{6 / 5}(I)$, in particular, each $\lambda_{j} \in W^{1, q}(I)$, for $q \in[1,6 / 5)$.

(2) Let $\left\{P_{\nu} ; \nu \in \mathcal{N}\right\}$ be a family of curves of polynomials (10.1) so that the set of coefficients $\left\{a_{\nu, j} ; \nu \in \mathcal{N}, j=1,2,3\right\}$ is bounded in $C^{6}(\bar{I})$. Then the set $\left\{\lambda_{\nu}^{\prime} ; \lambda_{\nu} \in\right.$ $C^{0}(I)$ with $P_{\nu}\left(\lambda_{\nu}\right)=0$ on $\left.I, \nu \in \mathcal{N}\right\}$ is bounded in $L_{w}^{6 / 5}(I)$.

Remark 10.2. In Theorem 10.1 it is actually enough to require that $f \in C^{5,1}(\bar{I}, \mathbb{C})$; cf. [7].

We sketch below the proof of Theorem 10.1. Thus consider $P$ after Tschirnhausen transformation

$$
P(Z)=Z^{3}+p Z+q
$$

The discriminant of $P$ equals

$$
\Delta=-27 q^{2}-4 p^{3}
$$

We assume that $p, q: I \rightarrow \mathbb{C}$ belong to $C^{6}(\bar{I})$. By Proposition 3.1 , for each $\delta \in[1 / 6,1)$,

$$
\begin{aligned}
& \left|p^{\prime}(t)\right| \leq \Lambda_{\delta^{-1}}(t)|p(t)|^{1-\delta} \\
& \left|q^{\prime}(t)\right| \leq \Lambda_{\delta^{-1}}(t)|q(t)|^{1-\delta} \quad \text { a.e. in } I \text { for some } \Lambda_{\delta^{-1}} \in L_{w}^{p}(I, \mathbb{R}), \text { where } p=\frac{1}{1-\delta} . \\
& \left|\Delta^{\prime}(t)\right| \leq \Lambda_{\delta^{-1}}(t)|\Delta(t)|^{1-\delta} \quad
\end{aligned}
$$

Here we set $\Lambda_{\delta^{-1}}:=\max \left\{\Lambda_{\delta^{-1}, p}, \Lambda_{\delta^{-1}, q}, \Lambda_{\delta^{-1}, \Delta}\right\}$. Note that each formula of (10.3) holds for every $t$ outside the zero set of $p, q$, or $\Delta$, respectively.

\section{Resolution of the Discriminant}

Consider the embedded resolution of the discriminant given by a sequence of three point blowing-ups. We denote it by $\sigma: M \rightarrow \mathbb{C}^{2}$. Note that $\sigma$ resolves also the ideal $\mathscr{I}=\left(p^{3}, q^{2}\right)$, that is, makes it locally principal and generated by a monomial. Thus in the notation of Section 1, $\sigma$ is a smooth principalization of $\mathscr{D}_{2}$ and $\mathscr{D}_{3}$ at the same time. Moreover, in this case, the formulas are explicit and we do not have to use the ideal $\mathscr{K}$ of Subsection 1.3 .

We describe the pull-back of $P$ by $\sigma$ in the affine charts and in each chart we give a formula for the roots of $P$. These formulas give the bounds on the derivative of the roots with respect to $t$. 
11.1. Chart 1. $p=X Y, q=Y$. This is one of two standard charts of the blowing-up of the origin. Then $\sigma^{*} \mathscr{I}=\left(Y^{2}\right)$ and

$$
\sigma^{*} P(Z)=Y\left(\tilde{Z}^{3}+X Y^{1 / 3} \tilde{Z}+1\right)=Y \tilde{P}(\tilde{Z}),
$$

where $Z=Y^{1 / 3} \tilde{Z}$. The polynomial $\tilde{P}$ has distinct roots near the exceptional divisor $Y=0$. Therefore by the Implicit Function Theorem (IFT) near the exceptional divisor the roots are of the form

$$
Z=Y^{1 / 3} \Phi\left(X Y^{1 / 3}\right)=q^{1 / 3} \Phi\left(p / q^{2 / 3}\right),
$$

where $\Phi$ is an analytic function (given locally by a convergent power series). Hence by (10.3)

$$
\left|Z^{\prime}(t)\right| \leq \frac{\left|q^{\prime}\right|}{|q|^{2 / 3}}|\Phi|+\frac{\left|p^{\prime}\right|}{|q|^{1 / 3}}\left|\Phi^{\prime}\right|+\frac{\left|p q^{\prime}\right|}{|q|^{4 / 3}}\left|\Phi^{\prime}\right| \leq \Lambda_{3}(t)\left(|\Phi|+C\left|\Phi^{\prime}\right|\right)
$$

taking into account that $p / q=X$ is bounded.

11.2. Chart 2. We take the other standard chart of the blowing-up of the origin $p=x, q=$ $x y$. The pull-back of the discriminant is not normal crossing in this chart and we have to blow up the origin again.

11.3. Chart 2a. $p=X, q=X^{2} Y$. This is one of the standard charts of the blowing-up of the origin of Chart 2: $x=X, y=X Y$. Then $\sigma^{*} \mathscr{I}=\left(X^{3}\right)$ and

$$
\sigma^{*} P(Z)=X^{3 / 2}\left(\tilde{Z}^{3}+\tilde{Z}+X^{1 / 2} Y\right)=X^{3 / 2} \tilde{P}(\tilde{Z})
$$

where $Z=X^{1 / 2} \tilde{Z}$. The discriminant of $\tilde{P}$ equals $-\left(4+27 X Y^{2}\right)$ and is non-zero near the exceptional divisor. Therefore by the IFT the roots of $\tilde{P}$ are convergent powers series in $X^{1 / 2} Y$ and hence the roots of $P$ are of the form

$$
Z=X^{1 / 2} \Phi\left(X^{1 / 2} Y\right)=p^{1 / 2} \Phi\left(q / p^{3 / 2}\right) .
$$

Hence by (10.3)

$$
\left|Z^{\prime}(t)\right| \leq \frac{\left|p^{\prime}\right|}{2|p|^{1 / 2}}|\Phi|+\frac{\left|q^{\prime}\right|}{|p|}\left|\Phi^{\prime}\right|+\frac{\left|q p^{\prime}\right|}{|p|^{2}}\left|\Phi^{\prime}\right| \leq \frac{1}{2} \Lambda_{2}(t)\left(|\Phi|+C\left|\Phi^{\prime}\right|\right)
$$

taking into account that $q / p^{2}=Y$ is bounded.

11.4. Chart 2b. $p=X Y, q=X Y^{2}$. The Chart $2 \mathrm{~b}$ is the other standard affine chart obtained by blowing up the origin in Chart 2: $x=x^{\prime} y^{\prime}, y=y^{\prime}$. Again the discriminant is not normal crossing in this chart so we have to blow-up again.

11.5. Chart $2 \mathbf{b}(\mathbf{i}) \cdot p=X^{2} Y, q=X^{3} Y^{2}$. This is one of the standard charts of blowing up the origin on Chart $2 \mathrm{~b}$, the other one is $2 \mathrm{~b}(\mathrm{ii})$. On Chart $2 \mathrm{~b}(\mathrm{i}), \sigma^{*} \mathscr{I}=\left(X^{6} Y^{3}\right)$ and $\Delta \circ \sigma=X^{6} Y^{3}(-4-27 Y)$. Then

$$
\sigma^{*} P(Z)=X^{3} Y^{3 / 2}\left(\tilde{Z}^{3}+\tilde{Z}+Y^{1 / 2}\right)=X^{3} Y^{3 / 2} \tilde{P}(\tilde{Z}),
$$

where $Z=X Y^{1 / 2} \tilde{Z}$. On the set where the discriminant of $\tilde{P}=-(4+27 Y)$ is non-zero we may again use the IFT. Then the roots of $P$ are of the form

$$
Z=X Y^{1 / 2} \Phi\left(Y^{1 / 2}\right)=p^{1 / 2} \Phi\left(q / p^{3 / 2}\right),
$$


where $\Phi$ is a convergent power series, as on Chart 2a. Now

$$
\begin{aligned}
\left|Z^{\prime}(t)\right| & \leq \frac{1}{2} \frac{\left|p^{\prime}\right|}{|p|^{1 / 2}}|\Phi|+\frac{\left|q^{2 / 3}\right|}{|p|} \frac{\left|q^{\prime}\right|}{\left|q^{2 / 3}\right|}\left|\Phi^{\prime}\right|+\frac{3}{2} \frac{|q|}{\left|p^{3 / 2}\right|} \frac{\left|p^{\prime}\right|}{|p|^{1 / 2}}\left|\Phi^{\prime}\right| \\
& \leq \frac{1}{2} \Lambda_{2}(t)|\Phi|+C\left(\Lambda_{3}(t)+\frac{3}{2} \Lambda_{2}(t)\right)\left|\Phi^{\prime}\right| .
\end{aligned}
$$

Near $Y=-\frac{4}{27}$ we introduce a new system of coordinates $\tilde{X}=X, \tilde{Y}=Y+\frac{4}{27}=\frac{q^{2}}{p^{3}}+\frac{4}{27}=$ $\frac{27 q^{2}+4 p^{3}}{27 p^{3}}$. Then $Y^{1 / 2}$ is a convergent power series in $\tilde{Y}$. At $\tilde{X}=\tilde{Y}=0$ the polynomial $\tilde{P}$ has one single and one double root. Therefore, by Lemma 9.1 we can factorize locally

$$
\tilde{P}(\tilde{Z})=\left(\tilde{Z}^{2}+b_{1}(\tilde{Y}) \tilde{Z}+b_{2}(\tilde{Y})\right)(\tilde{Z}+c(\tilde{Y})),
$$

where $b_{1}, b_{2}$ and $c=-b_{1}$ are convergent power series in $\tilde{Y}$. Thus one root of $P$ equals $-X Y^{1 / 2} c(\tilde{Y})$ and hence it can be written in the form

$$
Z=X \Phi(\tilde{Y})=\frac{p^{2}}{q} \Phi\left(\frac{q^{2}}{p^{3}}\right)=p^{1 / 2} \frac{p^{3 / 2}}{q} \Phi\left(\frac{q^{2}}{p^{3}}\right)=q^{1 / 3} \frac{p^{2}}{q^{4 / 3}} \Phi\left(\frac{q^{2}}{p^{3}}\right),
$$

where $\Phi$ is a convergent power series. Then, taking into account that $p^{3} \sim q^{2}$ near $\tilde{X}=\tilde{Y}=0$

$$
\begin{aligned}
\left|Z^{\prime}(t)\right| & \leq \frac{2\left|p p^{\prime}\right|}{|q|}|\Phi|+\frac{\left|p^{2} q^{\prime}\right|}{\left|q^{2}\right|}|\Phi|+3 \frac{|q|}{\left|p^{3 / 2}\right|} \frac{\left|p^{\prime}\right|}{\left|p^{1 / 2}\right|}\left|\Phi^{\prime}\right|+2 \frac{\left|q^{2 / 3}\right|}{|p|} \frac{\left|q^{\prime}\right|}{\left|q^{2 / 3}\right|}\left|\Phi^{\prime}\right| \\
& \leq C\left(\Lambda_{2}(t)+\Lambda_{3}(t)\right)|\Phi|+C\left(3 \Lambda_{2}(t)+2 \Lambda_{3}(t)\right)\left|\Phi^{\prime}\right|
\end{aligned}
$$

Denote the factors of (11.6) by $P_{b}$ and $P_{c}$. The discriminant of $P_{b}$ is the product of the discriminant of $\tilde{P}$ that is $-27 \tilde{Y}$, and an invertible convergent power series in $\tilde{Y}$. Indeed, this follows from the fact that the discriminant of $\tilde{P}$ equals the discriminant of $P_{b}$ times the square of the resultant of $P_{b}$ and $P_{c}$ which is invertible.

Let $\tilde{P}_{b}=Z^{2}+\tilde{b}_{2}$ be the Tschirnhausen transformation of $P_{b}$. The roots of $\tilde{P}_{b}$ equal, up to a constant, the square root of the discriminant of $P_{b}$. This gives the following form for the remaining two roots of $P$

$$
Z=Z_{0} \pm Z_{1}
$$

where $Z_{0}=-\frac{1}{2} X \Phi(\tilde{Y})$ is coming from (11.7), and $Z_{1}$ is of the form

$$
Z_{1}=X \tilde{Y}^{1 / 2} \Phi_{1}(\tilde{Y})=\frac{p^{1 / 2} \Delta^{1 / 2}}{q} \Phi_{1}\left(\frac{q^{2}}{p^{3}}\right)
$$

It remains to give a bound for $Z_{1}^{\prime}(t)$,

$$
\begin{aligned}
\left|Z_{1}^{\prime}(t)\right| \leq & \left(\frac{\left|p^{\prime}\right|}{\left|p^{1 / 2}\right|} \frac{\left|\Delta^{1 / 2}\right|}{|q|}+\frac{\left|p^{1 / 2}\right|}{\left|q^{1 / 3}\right|} \frac{\left|\Delta^{1 / 3}\right|}{\left|q^{2 / 3}\right|} \frac{\left|\Delta^{\prime}\right|}{\left|\Delta^{5 / 6}\right|}+\frac{\left|q^{\prime}\right|}{\left|q^{2 / 3}\right|} \frac{\left|p^{1 / 2}\right|}{\left|q^{1 / 3}\right|} \frac{\left|\Delta^{1 / 2}\right|}{|q|}\right)\left|\Phi_{1}\right| \\
& +\frac{\left|\Delta^{1 / 2}\right|}{|q|}\left(2 \frac{\left|q^{2 / 3}\right|}{|p|} \frac{\left|q^{\prime}\right|}{\left|q^{2 / 3}\right|}+3 \frac{\left|q^{2}\right|}{\left|p^{3}\right|} \frac{\left|p^{\prime}\right|}{\left|p^{1 / 2}\right|}\right)\left|\Phi_{1}^{\prime}\right| \\
\leq & C\left(\Lambda_{2}(t)+\Lambda_{6}(t)+\Lambda_{3}(t)\right)\left|\Phi_{1}\right|+C\left(2 \Lambda_{3}(t)+3 \Lambda_{2}(t)\right)\left|\Phi_{1}^{\prime}\right| .
\end{aligned}
$$

We have used that $p^{3} \sim q^{2}$ and that $\Delta / q^{2}$ is bounded near $\tilde{X}=\tilde{Y}=0$. 
11.6. Chart 2b(ii). $p=X Y^{2}, q=X Y^{3}$. Then $\sigma^{*} \mathscr{I}=\left(X^{2} Y^{6}\right)$ and $\Delta \circ \sigma=X^{2} Y^{6}(-4 X-$ 27). We only consider the points near the origin. The other points on this chart, including the strict transform of the discriminant, are also on Chart $2 \mathrm{~b}(\mathrm{i})$ and were considered before. On Chart 2b(ii)

$$
\sigma^{*} P(Z)=X Y^{3}\left(\tilde{Z}^{3}+X^{1 / 3} \tilde{Z}+1\right)=X Y^{3} \tilde{P}(\tilde{Z})
$$

where $Z=X^{1 / 3} Y \tilde{Z}$. Since $\tilde{P}$ has distinct roots near $X=Y=0$, by the IFT, the roots are of the form

$$
Z=X^{1 / 3} Y \Phi\left(X^{1 / 3}\right)=q^{1 / 3} \Phi\left(p / q^{2 / 3}\right)
$$

as on Chart 1. Then

$$
\left|Z^{\prime}(t)\right| \leq \frac{\left|q^{\prime}\right|}{|q|^{2 / 3}}|\Phi|+\frac{\left|p^{\prime}\right|}{|q|^{1 / 3}}\left|\Phi^{\prime}\right|+\frac{\left|p q^{\prime}\right|}{|q|^{4 / 3}}\left|\Phi^{\prime}\right| \leq \Lambda_{3}(t)\left(|\Phi|+C\left|\Phi^{\prime}\right|\right)
$$

taking into account that $p / q^{2 / 3}=X$ is bounded.
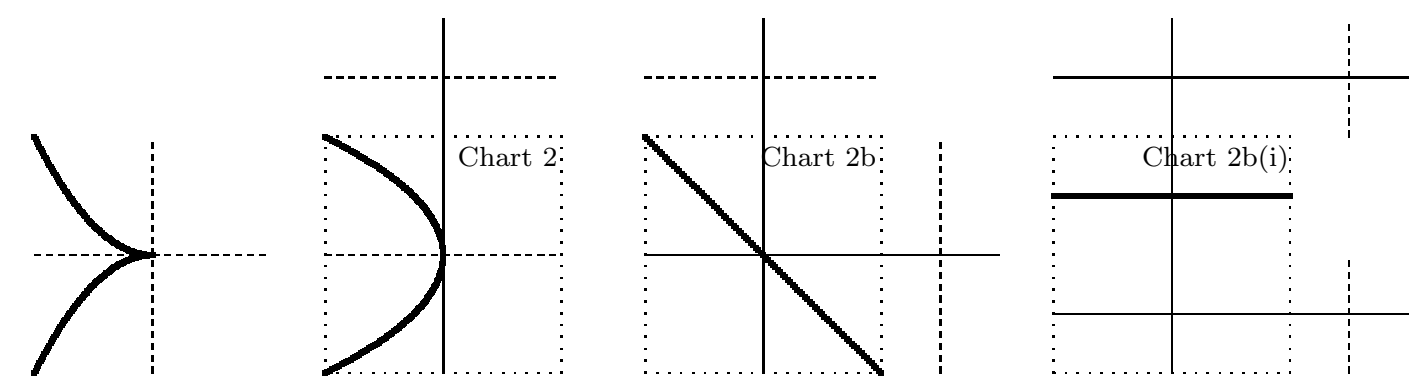

Figure 2. Bold curves represent the discriminant set and its strict transforms, thin (continuous) lines the exceptional divisors.

11.7. Proof of Theorem 10.1. The proof follows the reasoning of Subsection 3.5.

Let $p(t), q(t) \in C^{6}(\bar{I})$, let $\lambda(t)$ be a continuous root of (10.2), and let $\Omega=\{t \in I ;(p(t), q(t)) \neq$ $(0,0)\}$. Then $\left.(p(t), q(t))\right|_{\Omega}$ lifts to $M$ and Subsection 3.5 gives a bound on $\left\|\lambda^{\prime}\right\|_{6 / 5, w, \Omega}$. Since $\lambda \equiv 0$ on $I \backslash \Omega$, Theorem 10.1 follows from Lemma 2.1.

\section{REFERENCES}

[1] D. Alekseevsky, A. Kriegl, M. Losik, and P. W. Michor, Choosing roots of polynomials smoothly, Israel J. Math. 105 (1998), 203-233.

[2] E. Bierstone and P. D. Milman, Arc-analytic functions, Invent. Math. 101 (1990), no. 2, $411-424$.

[3] E. Bierstone and P. D. Milman, Canonical desingularization in characteristic zero by blowing up the maximum strata of a local invariant, Invent. Math. 128 (1997), 207-302.

[4] M. D. Bronshtein, Smoothness of roots of polynomials depending on parameters, Sibirsk. Mat. Zh. 20 (1979), no. 3, 493-501, 690, English transl. in Siberian Math. J. 20 (1980), 347-352.

[5] F. Colombini, E. Jannelli, and S. Spagnolo, Well-posedness in the Gevrey classes of the Cauchy problem for a nonstrictly hyperbolic equation with coefficients depending on time, Ann. Scuola Norm. Sup. Pisa Cl. Sci. (4) 10 (1983), no. 2, 291-312.

[6] F. Colombini and N. Lerner, Une procedure de Calderón-Zygmund pour le problème de la racine $k$-ième, Ann. Mat. Pura Appl. (4) 182 (2003), no. 2, 231-246.

[7] M. Ghisi and M. Gobbino, Higher order Glaeser inequalities and optimal regularity of roots of real functions, Ann. Sc. Norm. Super. Pisa Cl. Sci. (5) 12 (2013), no. 4, 1001-1021. 
[8] G. Glaeser, Racine carrée d'une fonction différentiable, Ann. Inst. Fourier (Grenoble) 13 (1963), no. 2, 203-210.

[9] L. Grafakos, Classical Fourier analysis, second ed., Graduate Texts in Mathematics, vol. 249, Springer, New York, 2008.

[10] R. Hartshorne, Algebraic geometry, Springer-Verlag, New York, 1977, Graduate Texts in Mathematics, No. 52.

[11] H. Hironaka, Resolution of singularities of an algebraic variety over a field of characteristic zero. I, II, Ann. of Math. (2) 79 (1964), 109-203; ibid. (2) 79 (1964), 205-326.

[12] T. Kato, Perturbation theory for linear operators, second ed., Grundlehren der Mathematischen Wissenschaften, vol. 132, Springer-Verlag, Berlin, 1976.

[13] J. Kollár, Lectures on resolution of singularities, Annals of Mathematics Studies, vol. 166, Princeton University Press, Princeton, NJ, 2007.

[14] A. Kriegl, M. Losik, P. W. Michor, and A. Rainer, Lifting smooth curves over invariants for representations of compact Lie groups. II, J. Lie Theory 15 (2005), no. 1, 227-234.

[15] G. Leoni, A first course in Sobolev spaces, Graduate Studies in Mathematics, vol. 105, American Mathematical Society, Providence, RI, 2009.

[16] B. Malgrange, Ideals of differentiable functions, Tata Institute of Fundamental Research Studies in Mathematics, No. 3, Tata Institute of Fundamental Research, Bombay, 1967.

[17] M. Marcus and V. J. Mizel, Absolute continuity on tracks and mappings of Sobolev spaces, Arch. Rational Mech. Anal. 45 (1972), 294-320.

[18] J. Milnor, Singular points of complex hypersurfaces, Annals of Mathematics Studies, No. 61, Princeton University Press, Princeton, N.J., 1968.

[19] A. Parusiński, G. Rond, The Abhyankar-Jung Theorem, Journal of Algebra 365 (2012), 29-41.

[20] A. Parusiński and A. Rainer, A new proof of Bronshtein's theorem, J. Hyperbolic Differ. Equ. 12 (2015), no. 4, 671-688.

[21] Q. I. Rahman and G. Schmeisser, Analytic theory of polynomials, London Mathematical Society Monographs. New Series, vol. 26, The Clarendon Press Oxford University Press, Oxford, 2002.

[22] A. Rainer, Perturbation of complex polynomials and normal operators, Math. Nachr. 282 (2009), no. 12, 1623-1636.

[23] A. Rainer, Quasianalytic multiparameter perturbation of polynomials and normal matrices, Trans. Amer. Math. Soc. 363 (2011), no. 9, 4945-4977.

[24] A. Rainer, Perturbation theory for normal operators, Trans. Amer. Math. Soc. 365 (2013), no. 10, 5545-5577.

[25] S. Spagnolo, On the absolute continuity of the roots of some algebraic equations, Ann. Univ. Ferrara Sez. VII (N.S.) 45 (1999), no. suppl., 327-337 (2000), Workshop on Partial Differential Equations (Ferrara, 1999).

[26] S. Spagnolo, Local and semi-global solvability for systems of non-principal type, Comm. Partial Differential Equations 25 (2000), no. 5-6, 1115-1141.

[27] S. Tarama, On the lemma of Colombini, Jannelli and Spagnolo, Memoirs of the Faculty of Engineering, Osaka City University 41 (2000), 111-115.

[28] S. Wakabayashi, Remarks on hyperbolic polynomials, Tsukuba J. Math. 10 (1986), no. 1, $17-28$.

Adam Parusiński: Univ. Nice Sophia Antipolis, CNRS, LJAD, UMR 7351, 06108 Nice, FRANCE

E-mail address: adam.parusinski@unice.fr

Armin Rainer: Fakultät für Mathematik, Universität Wien, Oskar-Morgenstern-Platz 1 , A-1090 Wien, Austria

E-mail address: armin.rainer@univie.ac.at 